\title{
Identification of the IncRNA-miRNA-mRNA network associated with gastric cancer via integrated bioinformatics analysis
}

\author{
XIAO-YU MA ${ }^{1}$, YU MA ${ }^{2}$, HUAN ZHOU ${ }^{1}$, HUI-JING ZHANG ${ }^{1}$ and MING-JUN SUN ${ }^{1}$ \\ Departments of ${ }^{1}$ Gastrointestinal Endoscopy and ${ }^{2}$ Nuclear Medicine, \\ First Affiliated Hospital of China Medical University, Shenyang, Liaoning 110001, P.R. China
}

Received December 26, 2018; Accepted July 26, 2019

DOI: $10.3892 / \mathrm{ol} .2019 .10922$

\begin{abstract}
The aim of the present study was to investigate the long non-coding RNA (lncRNA)-microRNA (miRNA)-mRNA regulatory network in gastric cancer (GC) using bioinformatics analysis. Two mRNA gene expression profiles, GSE79973 and GSE54129, and two miRNA expression profiles, GSE93415 and GSE78091, were downloaded from the Gene Expression Omnibus database. The differentially expressed mRNAs (DEMs) and the differentially expressed miRNAs (DEMis) were merged separately. Gene ontology and pathway enrichment analysis were conducted using the Database for Annotation, Visualization and Integrated Discovery. A protein-protein interaction (PPI) network was then constructed and the 10 top hub genes in the network were analyzed using the Search Tool for the Retrieval of Interacting Genes. The lncRNA-miRNA-mRNA networks were visualized using Cytoscape software. As a result, 158 shared DEMs (40 upregulated and 118 downregulated) were identified from two mRNA datasets. A total of 30 upregulated miRNAs and 1 downregulated miRNA functioned as DEMis. The PPI network
\end{abstract}

Correspondence to: Dr Ming-Jun Sun, Department of Gastrointestinal Endoscopy, First Affiliated Hospital of China Medical University, 155 North Nanjing Street, Shenyang, Liaoning 110001, P.R. China

E-mail: mingjunsun@126.com

Abbreviations: BP, biological processes; CC, cellular component; DAVID, Database for Annotation, Visualization and Integrated Discovery; DEM, differentially expressed mRNA; DEMi, differentially expressed miRNA; GC, gastric cancer; GEO, Gene Expression Omnibus; GO, Gene ontology; KEGG, Kyoto Encyclopedia of Genes and Genomes; lncRNA, long non-coding RNA; MF, molecular function; miRNA, microRNA; OS, overall survival; PPI, protein-protein interaction network; STRING, Search Tool for the Retrieval of Interacting Genes

Key words: gastric cancer, long non-coding RNA, microRNA, bioinformatics analysis, gene expression omnibus, protein-protein interaction network consisted of 129 nodes and 572 interactions. The 10 top hub genes were selected by degree using Cytohubba, including Jun proto-oncogene, mitogen-activated protein kinase (MAPK)3, transforming growth factor- $\beta 1$, Fos proto-oncogene, AP-1 transcription factor subunit, interleukin (IL)-8, MAPK1, RELA proto-oncogene nuclear factor- $\kappa \mathrm{B}$ subunit, interferon regulatory factor 7, ubiquitin like modifier and vascular endothelial growth factor A. In the IncRNA-miRNA-mRNA network, a total of 1,215 regulatory associations were constructed using Cytoscape. In conclusion, the present study provides a novel perspective of the molecular mechanisms underlying GC by identifying the IncRNA-miRNA-mRNA regulatory network via bioinformatics analysis.

\section{Introduction}

Gastric cancer (GC) is one of the most common malignant diseases globally (1). Although substantial advances have been made in the diagnosis and therapy of this disease, the prognosis of GC remains poor and the 5-year survival rate is still comparatively low $(2,3)$. Therefore, it is essential to investigate the molecular mechanism including potential biomarkers and therapeutic targets of GC.

In previous years, a large number of microarrays and bioinformatics methods have been conducted to investigate the molecular mechanism underlying cancer progression including diagnosis, treatment and prognosis (4-6). For example, bioinformatics analysis has been used to elucidate the potential key candidate genes and pathways in colorectal cancer from four cohort profile datasets (7). In addition, target genes and the prognostic value in non-small cell lung cancer have been discovered previously via bioinformatics analysis (8). Similarly, bioinformatics analysis has been performed to identify long non-coding RNA (lnc-RNA)-microRNA (miRNA/miR)-mRNA networks via the combination of lncRNA, miRNA and mRNA expression profiles based on competitive endogenous RNA in rheumatoid arthritis (9). In conclusion, it is necessary to perform further investigation of the molecular mechanism underlying GC using integrated bioinformatics analysis.

In the present study, differentially expressed mRNAs (DEMs) and differentially expressed miRNAs (DEMis) were 
screened out from the Gene Expression Omnibus (GEO) database. Gene Ontology (GO) and Kyoto Encyclopedia of Genes and Genomes (KEGG) pathway enrichment analyses of DEMs were also performed. The lncRNA-miRNA-mRNA network was subsequently established, which may provide additional information on the molecular mechanism of GC.

\section{Materials and methods}

Gene expression profiles. Two human mRNA expression profiles, GSE79973 and GSE54129, were acquired from the GEO database (ncbi.nlm.nih.gov/geo/) (10), which included $121 \mathrm{GC}$ and 31 normal samples. Two human miRNA expression profiles, GSE93415 and GSE78091, were also downloaded from the GEO database, which included $23 \mathrm{GC}$ and 23 normal samples. FunRich version 3.1.3 was used to draw the venn diagram (11).

Identification of DEMs and DEMis. Two mRNA and two miRNA databases were analyzed using the GEO2R web tool comparing samples in the GC and control groups (12). In order to select the DEMs, an adjusted (adj.) P-value of $\leq 0.05$ and $\mid \log 2$ fold change (FC) $\mid \geq 2$ were selected as the cut-off values for the two mRNA databases. For DEMis, adj.P-value $\leq 0.05$ and $\log 2 \mathrm{FCl} \geq 1$ were regarded as the cut-off criteria values.

Function analysis of DEMs. GO function and KEGG pathway enrichment analysis of the up- and downregulated genes and 10 hub genes were analyzed using the online Database for Annotation, Visualization and Integrated Discovery (DAVID; david.abcc.ncifcrf.gov/). $\mathrm{P}<0.05$ was considered to indicate a statistically signicant difference.

Protein-protein interaction (PPI) network construction and sub-network identification. Search Tool for the Retrieval of Interacting Genes (STRING; string-db.org/) was used to analyze the PPI network of DEMs (13). The PPIs of DEMs were selected using a combined score of $>0.9$. Cytoscape version 3.5.1 (cytoscape.org/) software was utilized to construct the PPI network (14). Cytohubba in Cytoscape software was employed to identify the 10 top hub genes by degree. The sub-network in the PPI network was then visualized by MCODE with a cut-off criterion of $\mathrm{k}$-score=2 (15). $\mathrm{P}<0.05$ was considered to indicate a statistically signicant difference.

Survival analysis of the 10 top hub genes. The publicly available database Kaplan-Meier Plotter (www.kmplot.com) was used to identify the prognostic effect of the 10 top hub genes (16). $\mathrm{P}<0.05$ was considered to indicate a statistically signicant difference.

Determining the expression level of the significantly up- and downregulated DEMs. A total of 5 significantly upregulated DEMs (inhibin bA, collagen type VIII $\alpha 1$, secreted frizzle related protein 4 , secreted phosphoprotein 1 and thormbospondin 2) and 5 significantly downregulated DEMs [Gastrokine 2 (GKN2), GKN1, gastric intrinsic factor, Mucin like 3 and Keratin 20 (KRT20)] were selected for further analysis. The Gene Expression Profiling Interactive Analysis
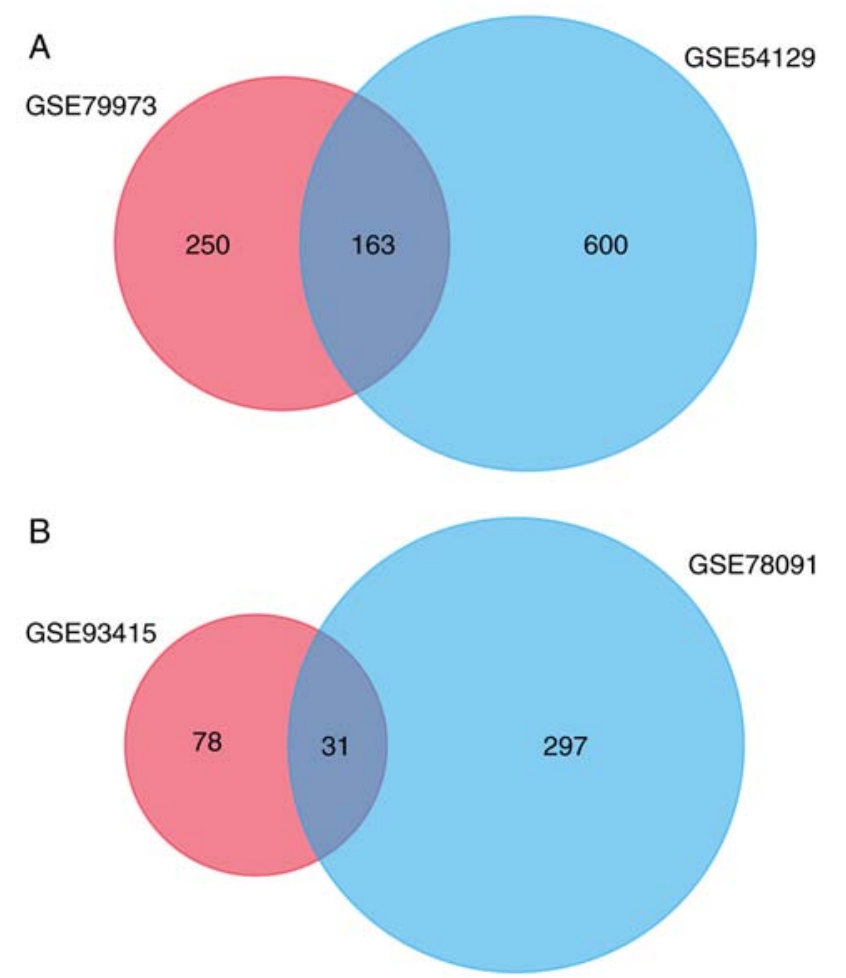

Figure 1. Identification of DEMs and DEMis. (A) A Venn diagram of DEMs in GSE79973 and GSE54129 produced using FunRich version 3.1.3. (B) A Venn diagram of DEMis in GSE93415 and GSE78091. DEM, differentially expressed mRNA; DEMis, differentially expressed microRNA.

(GEPIA; gepia.cancer-pku.cn/) database was used to determine the expression of 10 DEMs in GC tissues (17). In order to provide more sufficient evidence to support the results, the present study investigated the data in the Cancer Cell Line Encyclopedia (CCLE) portals (broadinstitute.org/ccle/about) database, which supplied information on the expression of 10 DEMs in GC cell lines (18). $\mathrm{P}<0.05$ was considered to indicate a statistically signicant difference.

Reconstruction of the IncRNA-miRNA-mRNA networks. The miRNA-targeted genes were screened out using the miRWalk database (mirwalk.umm.uni-heidelberg.de/) with a score of $>0.9(19,20)$. The targeted genes and DEMs mentioned above were merged to identify the number of miRNA-regulated target gene pairs. The prediction of IncRNA-miRNA interactions was based on the analysis of the IncRNASNP (www. lncRNAblog.com) database (21). The networks between miRNA-mRNA and IncRNA-miRNA were visualized using Cytoscape version 3.5.1. $\mathrm{P}<0.05$ was considered to indicate a statistically signicant difference.

Statistical analysis. All statistical analysis was performed using corresponding databases. Data was presented as the mean \pm standard deviation. A paired Student's t-test or one-way analysis of variance (ANOVA) or two-way ANOVA were used to evaluate the degree of differential expression. A log-rank test was applied to analyse the association between expression and prognosis. Post-hoc tests (least significant difference and Tukey's tests) were performed following ANOVA. $\mathrm{P}<0.05$ was considered to indicate a statistically significant difference. 
Table I. 158 DEMs were obtained from two datasets, including 40 upregulated DEMs and 118 downregulated DEMs. 31 DEMis were obtained between gastric cancer samples and control samples, including 30 upregulated miRNAs and 1 downregulated miRNA.

DEMs

Gene name

Upregulated

COL11A1, INHBA, IGF2BP3, COL10A1, FNDC1, FAP, THBS2, SULF1, CST1, COL8A1, SFRP4, SPP1, COL1A1, ADAMTS2, WISP1, CTHRC1, COL12A1, ASPN, CRISPLD1, THY1, COL1A2, FN1, BGN, RARRES1, CAP2, MFAP2, PDPN, PRRX1, TIMP1, SPARC, COL6A3, COL4A1, THBS1, NRP2, PDLIM7, LY6E, SPOCK1, PI15, CEMIP, CXCL8

Downregulated GKN2, GKN1, ATP4A, ATP4B, GIF, LIPF, KCNJ16, DPCR1, SOSTDC1 KCNE2, CWH43, ESRRG, PGC, SLC28A2, PSAPL1, KRT20, VSIG1, LTF, CXCL17, AKR1B10, LOC643201, GSTA1, ADH7, ADH1C, GC, CAPN9, MAL, SLC26A9, HRASLS2, MFSD4A, MUC5AC, FBP2, ALDH3A1, ADGRG2, VSTM2A, LINC00982, CAPN13, KIAA1324, CA9, TPCN2, PIK3C2G, RDH12, SLC26A7, SSTR1, VSIG2, GATA6-AS1, HPGD, UPK1B, KCNJ15, SULT1C2, LINC00675, BTNL8, AXDND1, LINC00992, KAZALD1, TMED6, UGT2B15, SCNN1B, HAPLN1, AKR1C1, LYPD6B, FCGBP, ADTRP, IGH, CA2, RFX6, ACER2, CYP2C9, PCAT18, PKIB, SH3RF2, HHIP, HEPACAM2, AADAC, CYP2C18, RAB27B, MGAM, SPINK7, CNTN3, LINC01133, BCAS1, SULT1B1, CAPN8, SMIM6, AMPD1, JCHAIN, PBLD, ATP13A4, RNASE1, PLLP, B4GALNT3, STYK1, CYP2C19, SMIM24, LRRC66, RASSF6, ADAM28, FA2H, GATA5, SCIN, SGK2, TPH1, PROM2, APOBEC1, ACKR4, ADH1A, AKR7A3, OASL, SMPD3, XK, KLHDC7A, STX19, CYP3A5, STS, VILL, ANG, S100P, DDX60

DEMis

miRNA name

Upregulated

hsa-let-7i-3p, hsa-miR-100-5p, hsa-miR-106b-5p, hsa-miR-10a-5p, hsa-miR-151a-5p, hsa-miR-15a-5p, hsa-miR-195-5p, hsa-miR-199a-3p, hsa-miR-199a-5p, hsa-miR-199b-5p, hsa-miR-19a-3p, hsa-miR-20a-5p, hsa-miR-214-3p, hsa-miR-214-5p, hsa-miR-218-5p, hsa-miR-223-3p, hsa-miR-301a-3p, hsa-miR-331-3p, hsa-miR-335-5p, hsa-miR-342-3p, hsa-miR-377-3p, hsa-miR-4262, hsa-miR-4291, hsa-miR-4317, hsa-miR-454-3p, hsa-miR-455-3p, hsa-miR-4791, hsa-miR-93-5p, hsa-miR-99a-5p, hsa-miR-99b-5p

Downregulated hsa-miR-375

DEM, differentially expressed mRNA; DEMis, differentially expressed microRNA; miRNA/miR, microRNA.

\section{Results}

Identification of DEMs and DEMis. A total of 158 DEMs (5 of the 163 genes were identified as unreconized genes) were identified based on the cut-off criteria using FunRich, including 40 upregulated DEMs and 118 downregulated DEMs (Fig. 1A). Furthermore, 31 DEMis were obtained between GC and control samples. A total of 30 upregulated miRNAs and 1 downregulated miRNA were identified as DEMis (Fig. 1B). All DEMs and DEMis are presented in Table I.

Functional analysis of DEMs. According to GO functional enrichment analysis for up- and downregulated DEMs, the significantly enriched biological process (BP), cellular component (CC) and molecular function (MF) terms were selected (Fig. 2). The values in the $\mathrm{X}$-axes represent the quantification of $-\log 10$ (P-value). The GO terms enriched by upregulated DEMs were mainly associated with 'endodermal cell differentiation', 'proteinaceous extracellular' and 'extracellular matrix binding' while the GO terms enriched by downregulated DEMs were mainly associated with 'regulation of cell proliferation', 'extracellular exosome' and 'iron ion binding' (Table II). Following KEGG pathway enrichment analysis, upregulated differentially expressed genes (DEGs) were revealed to be mainly involved in 'ECM-receptor interaction', 'focal adhe- sion' and 'phosphoinositide-3-kinase (PI3K)-protein kinase B (Akt) signaling pathway'. Downregulated DEGs were associated with 'gastric acid secretion', 'retinol metabolism' and 'chemical carcinogenesis' (Table III).

PPI network construction and sub-network identification. The PPI network consisted of 129 nodes and 572 interactions (Fig. 3). Following the use of Cytohubba in Cytoscape software, 10 top hub genes Jun proto-oncogene (JUN), mitogen activated protein kinase (MAPK)3, transforming growth factor- $\beta 1$, Fos proto-oncogene, AP-1 transcription factor subunit (FOS), interleukin (IL)-8, MAPK1, RELA proto-oncogene nuclear factor $\kappa \beta(N F-\kappa B)$ subunit (RELA), interferon regulatory factor 7 (IRF7), ubiquitin like modifier (ISG15) and vascular endothelial growth factor A (VEGFA) were evaluated by degree in the PPI network (Fig. 4). The sub-network in the PPI network was then visualized using MCODE with a cut-off criterion of $k$-score $=2$. The sub-network was obtained from the PPI network with 14 nodes and 86 interactions (Fig. 5).

Survival analysis of the 10 top hub genes. The present study used the Kaplan-Meier Plotter database to evaluate the prognostic effect of the 10 top hub genes by overall survival (OS; Fig. 6). The Kaplan-Meier curves indicated that a higher expression of MAPK3, TGFB1, RELA, IRF7, ISG15 and 

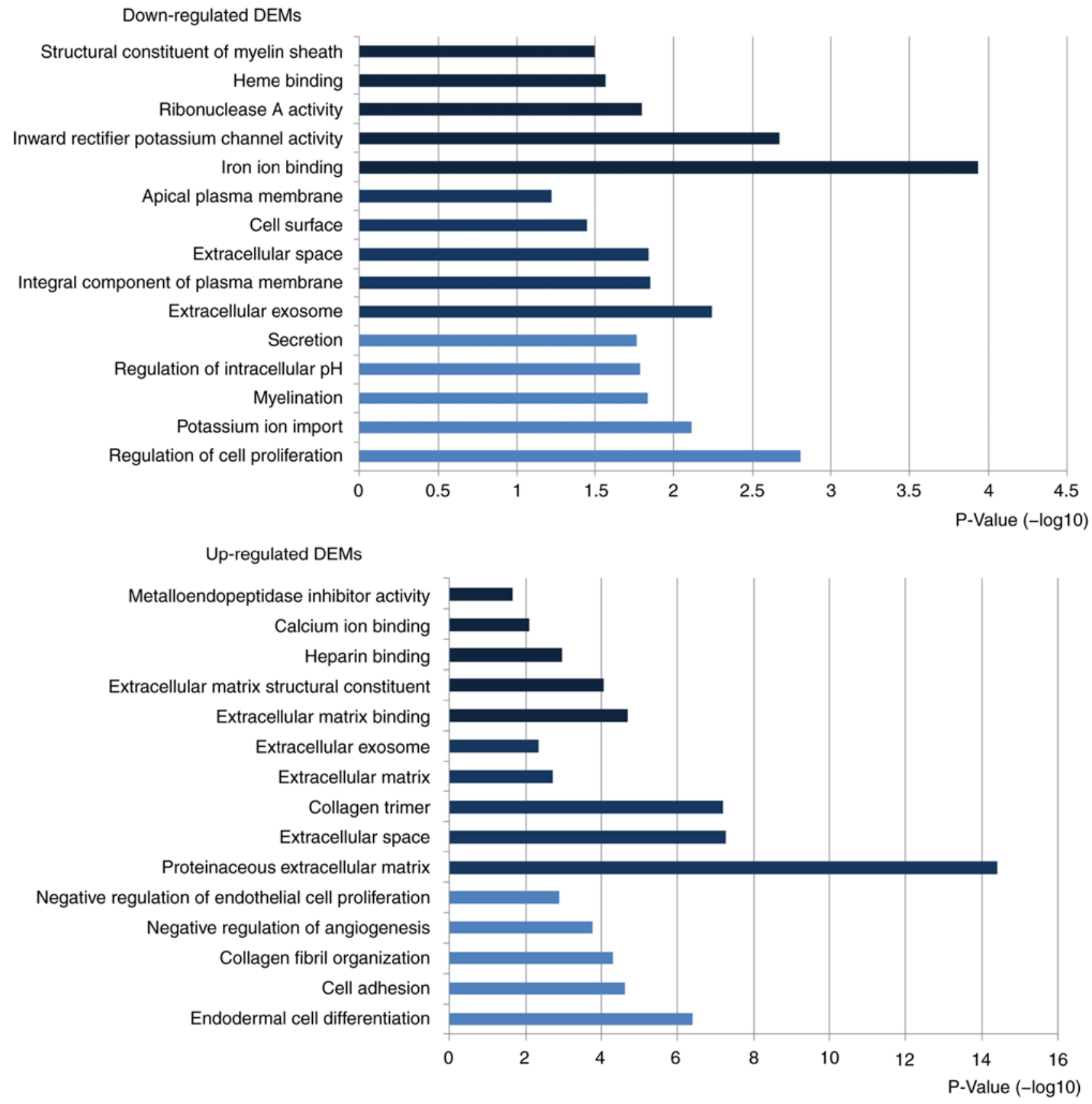

Figure 2. Top 5 enriched Gene Ontology terms of upregulated and downregulated DEMs. [Black represents biological processes, dark blue indicates cellular component, and light blue denotes molecular function; - Log10 (P-value) represents the value of $x$-axes]. P $<0.05$. DEM, differentially expressed mRNA.

VEGFA was significantly associated with poor survival in GC. On the other hand, a lower expression of JUN, FOS, IL8 and MAPK1 was significantly associated with poor survival in GC.

Functional analysis of 10 top hub genes and DEMs. In order to elucidate the specific signaling pathways that the $158 \mathrm{DEMs}$ and 10 top hub genes were involved in, the DAVID database was used for further research. The results revealed that the 158 DEMs were involved in 17 KEGG pathways (Table IV). Among these pathways, 4 KEGG pathways (bta04151: PI3K-Akt signaling pathway, bta04510: Focal adhesion, bta05146: Amoebiasis and bta05144: Malaria) were identi- fied to be significantly associated with the 10 top hub genes (Table V).

Expression levels of significantly up-and downregulated $D E M s$. The GEPIA database was used to reveal the expression levels of 10 DEMs in GC tissues. The results revealed that 9 DEMs presented the same trend that was previously noted and only 1 DEM (KRT20) was contrary to the aforementioned trend (Fig. 7). The CCLE database was used to obtain information on the expression levels of 10 DEMs in GC cell lines. The present study then selected 5 different GC cell lines (HGC27, HS746T, MKN1, NUGC3 and RERFGC1B) to analyze the expression levels of the 10 
Table II. Top 5 enriched GO terms of upregulated and downregulated differentially expressed mRNAs.

\begin{tabular}{|c|c|c|c|}
\hline Upregulated & Term & Count & P-value \\
\hline \multicolumn{4}{|l|}{$\mathrm{BP}$} \\
\hline GO:0035987 & Endodermal cell differentiation & 5 & $4.00 \times 10^{-07}$ \\
\hline GO:0007155 & Cell adhesion & 6 & $2.44 \times 10^{-05}$ \\
\hline GO:0030199 & Collagen fibril organization & 4 & $5.04 \times 10^{-05}$ \\
\hline GO:0016525 & Negative regulation of angiogenesis & 4 & $1.66 \times 10^{-04}$ \\
\hline GO:0001937 & Negative regulation of endothelial cell proliferation & 3 & 0.001248824 \\
\hline \multicolumn{4}{|l|}{$\mathrm{CC}$} \\
\hline GO:0005578 & Proteinaceous extracellular matrix & 13 & $4.06 \times 10^{-15}$ \\
\hline GO:0005615 & Extracellular space & 14 & $5.60 \times 10^{-08}$ \\
\hline GO:0005581 & Collagen trimer & 6 & $6.88 \times 10^{-08}$ \\
\hline GO:0031012 & Extracellular matrix & 4 & 0.002041798 \\
\hline GO:0070062 & Extracellular exosome & 13 & 0.004832505 \\
\hline \multicolumn{4}{|l|}{ MF } \\
\hline GO:0050840 & Extracellular matrix binding & 4 & $2.15 \times 10^{-05}$ \\
\hline GO:0005201 & Extracellular matrix structural constituent & 4 & $9.22 \times 10^{-05}$ \\
\hline GO:0008201 & Heparin binding & 4 & 0.001122327 \\
\hline GO:0005509 & Calcium ion binding & 6 & 0.008415225 \\
\hline GO:0008191 & Metalloendopeptidase inhibitor activity & 2 & 0.02384997 \\
\hline \multicolumn{4}{|l|}{ Downregulated } \\
\hline \multicolumn{4}{|l|}{$\mathrm{BP}$} \\
\hline GO:0042127 & Regulation of cell proliferation & 6 & 0.001561961 \\
\hline GO:0010107 & Potassium ion import & 3 & 0.007733402 \\
\hline GO:0042552 & Myelination & 3 & 0.014660727 \\
\hline GO:0051453 & Regulation of intracellular $\mathrm{pH}$ & 3 & 0.01645907 \\
\hline GO:0046903 & Secretion & 2 & 0.017262077 \\
\hline \multicolumn{4}{|l|}{$\mathrm{CC}$} \\
\hline GO:0070062 & Extracellular exosome & 22 & 0.00577329 \\
\hline GO:0005887 & Integral component of plasma membrane & 11 & 0.014280261 \\
\hline GO:0005615 & Extracellular space & 12 & 0.014702585 \\
\hline GO:0009986 & Cell surface & 6 & 0.036068461 \\
\hline GO:0016324 & Apical plasma membrane & 4 & 0.06075832 \\
\hline \multicolumn{4}{|l|}{ MF } \\
\hline GO:0005506 & Iron ion binding & 7 & $1.17 \times 10^{-04}$ \\
\hline GO:0005242 & Inward rectifier potassium channel activity & 3 & 0.002175164 \\
\hline GO:0004522 & Ribonuclease A activity & 2 & 0.016178947 \\
\hline GO:0020037 & Heme binding & 4 & 0.027324696 \\
\hline GO:0019911 & Structural constituent of myelin sheath & 2 & 0.032099763 \\
\hline
\end{tabular}

GO, Gene Ontology; BP, biological processes; CC, cellular component; MF, molecular function.

DEMs (Fig. 8). For the upregulated DEMs, the expression level was partially different from the aforementioned results. All downregulated DEMs exhibited the same trend observed previously in the present study.

Association analysis of IncRNAs, miRNAs and mRNAs. The network of miRNAs and their targets was composed of 85 nodes and 145 interactions, determined using Cytoscape software (Fig. 9). In the IncRNA-miRNA-mRNA network, a total of 1,215 regulatory associations were screened out (Fig. 10). Pink circles indicated lncRNAs, hexagons indicated
miRNAs, and diamonds represented mRNAs. With regards to the colors, red nodes indicated upregulated mRNAs and miRNAs, and green nodes represented downregulated mRNAs and miRNAs.

\section{Discussion}

The identification of the underlying molecular mechanism of GC is necessary to detect therapeutic targets in the malignant transformation process for management strategies. To date, microarrays and bioinformatics methods have been 
Table III. Top 5 enriched Kyoto Encyclopedia of Genes and Genomes pathway analysis of upregulated and downregulated differentially expressed mRNAs.

\begin{tabular}{llrr}
\hline ID & \multicolumn{1}{c}{ Term } & Count & P-value \\
\hline $\begin{array}{l}\text { Upregulated } \\
\text { cfa04512 } \\
\text { cfa04510 }\end{array}$ & Extracellular matrix-receptor interaction & & \\
cfa04974 & Focal adhesion & 9 & $3.17 \times 10^{-12}$ \\
cfa04151 & Protein digestion and absorption & 9 & $3.53 \times 10^{-09}$ \\
cfa05146 & Phosphoinositide-3-kinase-protein kinase B signaling pathway & 7 & $1.38 \times 10^{-08}$ \\
\hline ID & Amoebiasis & 9 & $1.62 \times 10^{-07}$ \\
\hline Downregulated & Term & Count & P-value \\
bta04971 & & & \\
bta00830 & Gastric acid secretion & 7 & $1.29 \times 10^{-06}$ \\
bta05204 & Retinol metabolism & 6 & $8.34 \times 10^{-06}$ \\
bta01100 & Chemical carcinogenesis & 6 & $2.30 \times 10^{-05}$ \\
bta00140 & Metabolic pathways & 16 & $5.74 \times 10^{-04}$ \\
\hline
\end{tabular}

used to analyze the process of carcinogenesis to enhance the universality and reliability of the results (4-8). In the present study, 158 shared DEMs (40 upregulated and 118 downregulated) were obtained from the investigation of the GSE79973 and GSE54129 datasets. One previous study used GSE79973 to identify 14 significantly downregulated genes in GC, including KRT20, cytochrome P450 family 3 subfamily A member 5, RAB27B member RAS oncogene family and sulfotransferase family $1 \mathrm{C}$ member 2 (22). The four genes were additionally downregulated in the present study. Another previous study using GSE54129 identified 1829 DEMs including 838 upregulated genes and 991 downregulated genes (23). The Affy and limma packages in $\mathrm{R}$ software were used to select the DEMs in previous studies. In the present study, 158 shared DEMs (40 upregulated and 118 downregulated) were selected. The GEO2R web tool was used in the present study to identify DEMs. Although the data was from the same database, unequal results were obtained due to the different processing methods and filtering conditions utilized. For the analysis of 10 hub nodes, the previous study using the GSE54129 dataset identified 10 top hub nodes [tumor protein $\mathrm{p} 53, \mathrm{C}-\mathrm{X}-\mathrm{C}$ motif chemokine ligand (CXCL)8, tetraspanin 4, lysophospatidic acid receptor 2, adenylate cyclase 3, phosphoinositide-3-kinase regulatory subunit 1 , neuromedin U, CXCL1, FOS and sphingosine-1-phosphate receptor 1] (23). FOS was also one of 10 hub genes identified in the present study. Meanwhile, the present study performed GO function and KEGG pathway enrichment analyses with 158 DEGs. The significant GO terms enriched by upregulated DEMs included 'endodermal cell differentiation', 'proteinaceous extracellular' and 'extracellular matrix binding' while the terms enriched by downregulated DEMs were mainly associated with 'regulation of cell proliferation', 'extracellular exosome' and 'iron ion binding'. The results of KEGG pathway enrichment revealed that upregulated DEGs were mainly involved in 'ECM-receptor interaction', 'focal adhesion' and 'PI3K-Akt signaling pathway', and downregulated DEGs were associated with 'gastric acid secretion', 'retinol metabolism' and 'chemical carcinogenesis'. Among the enriched pathways, the 'PI3K/Akt signaling pathway' has an essential biological function in the development of proliferation, apoptosis and invasion in various types of human cancer, including GC (24-26). Furthermore, the PI3K/Akt signaling pathway is commonly activated in advanced GC and serves an important function in resistance to chemotherapy in GC (27). LY294002 has been identified as a PI3K inhibitor and has been confirmed to suppress cell proliferation and enhance apoptosis by downregulating VEGF, matrix metalloproteinase (MMP)-2 and MMP-9 in GC (28).

In the constructed PPI network, there were 129 nodes and 572 interactions. The top 10 hub genes (JUN, MAPK3, TGFB1, FOS, IL8, MAPK1, RELA, IRF7, ISG15 and VEGFA) were evaluated by degree following Cytohubba analysis. Then, the DAVID database was used to elucidate the specific signaling pathways that the 158 DEMs and 10 top hub genes were involved in. The results revealed that 158 DEMs were involved in 17 KEGG pathways (Table IV). Amongst these pathways, 4 KEGG pathways (bta04151: PI3K-Akt signaling pathway, bta04510: Focal adhesion, bta05146: Amoebiasis and bta05144: Malaria) were also associated with the 10 hub genes (Table V). Among four common KEGG pathways, PI3K-Akt signaling pathway is closely associated with the function of miRNAs. miR-15a-5p (an upregulated DEMi) regulated granulosa cell proliferation by activating the PI3K-Akt-mechanistic target of rapamycin kinase (mTOR) signaling pathway (29). miR-4262 (an upregulated DEMi) regulates chondrocyte viability, apoptosis, autophagy by targeting sirtuin 1 and activating the PI3K/Akt/mTOR signaling pathway in rats with osteoarthritis (30). miR-375 (a downregulated DEMi) functions as a tumor suppressor in osteosarcoma and colorectal cancer by targeting phosphatidylinositol-4,5-bisphosphate 


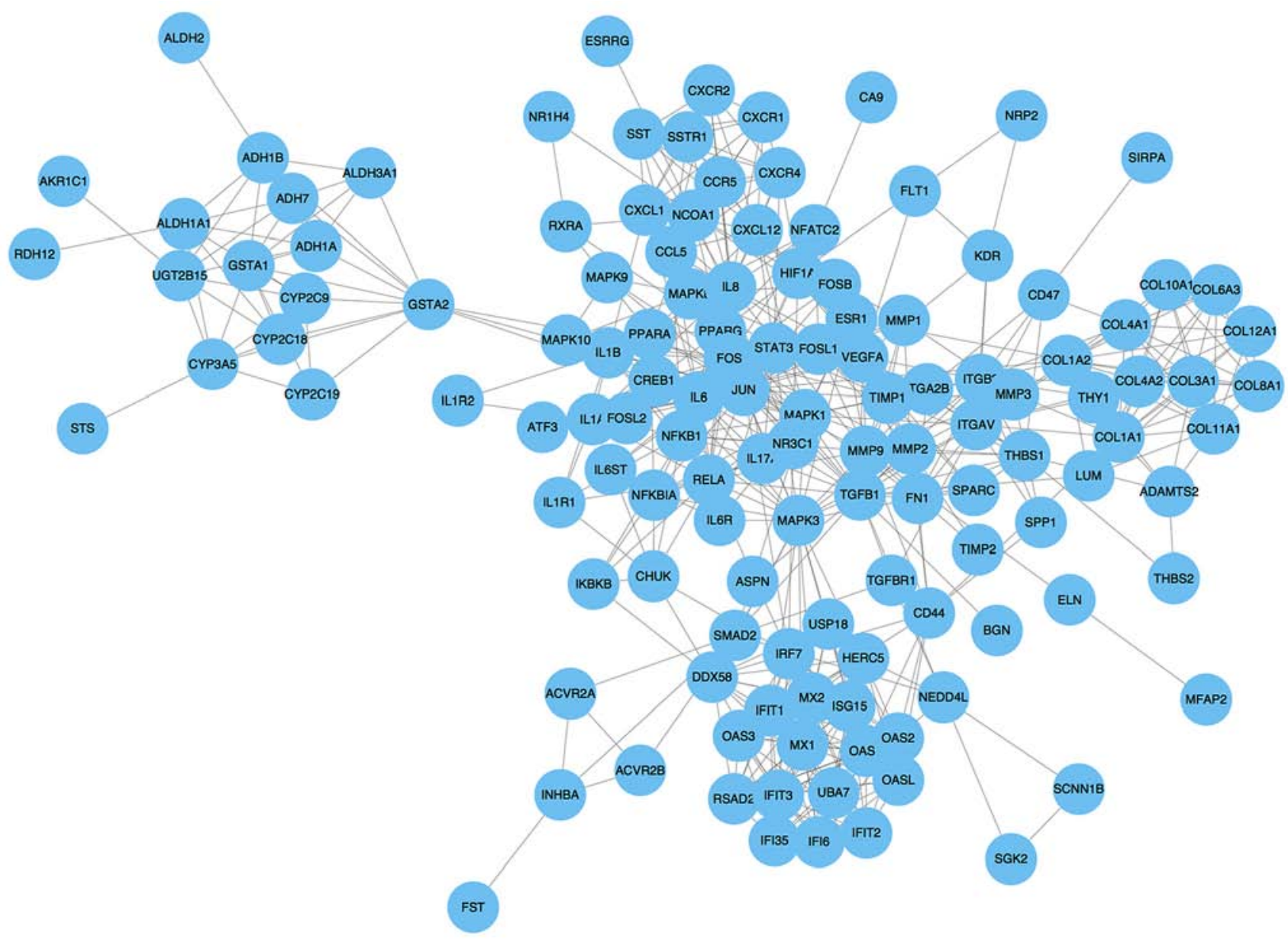

Figure 3. Construction of a protein-protein interaction network of differentially expressed mRNAs. $\mathrm{P}<0.05$.

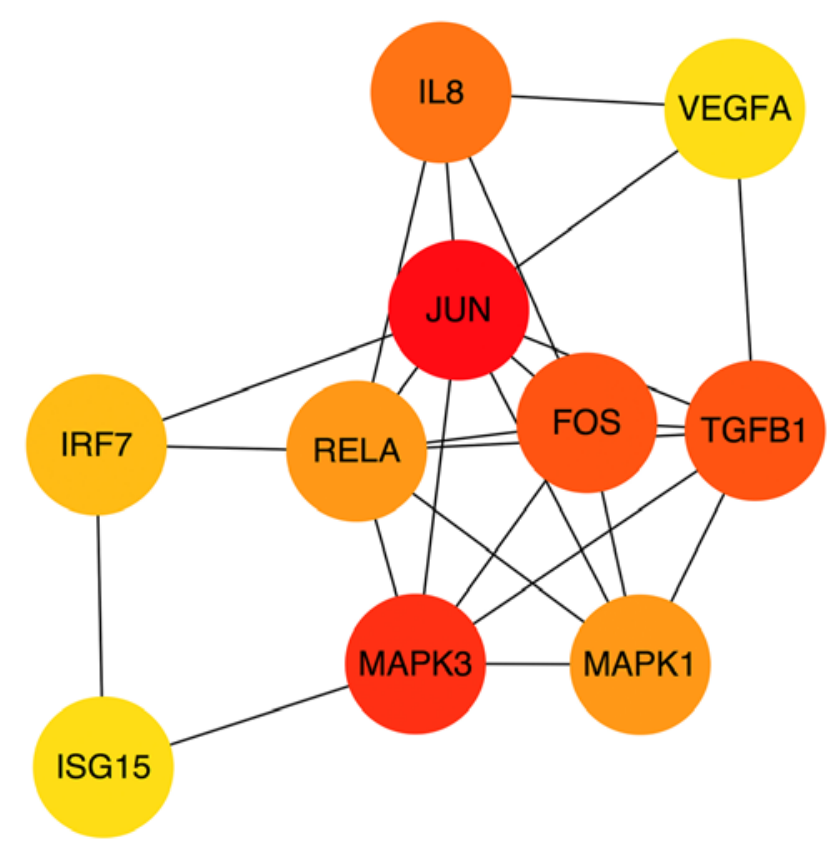

Figure 4. Top 10 hub genes by degree in the protein-protein interaction network using the Cytohubba in Cytoscape software. $\mathrm{P}<0.05$. JUN, Jun proto-oncogene; TGFB1, transforming growth factor- $\beta 1$; FOS, Fos proto-oncogene, AP-1 transcription factor subunit; IL-8, interleukin 8; MAPK, mitogen-activated protein kinase; RELA, RELA proto-oncogene NF-ein kinase; IRF7, interferon regulatory factor 7; VEGFA, vascular endothelial growth factor A; ISG15, ISG15 ubiquitin like modifier.

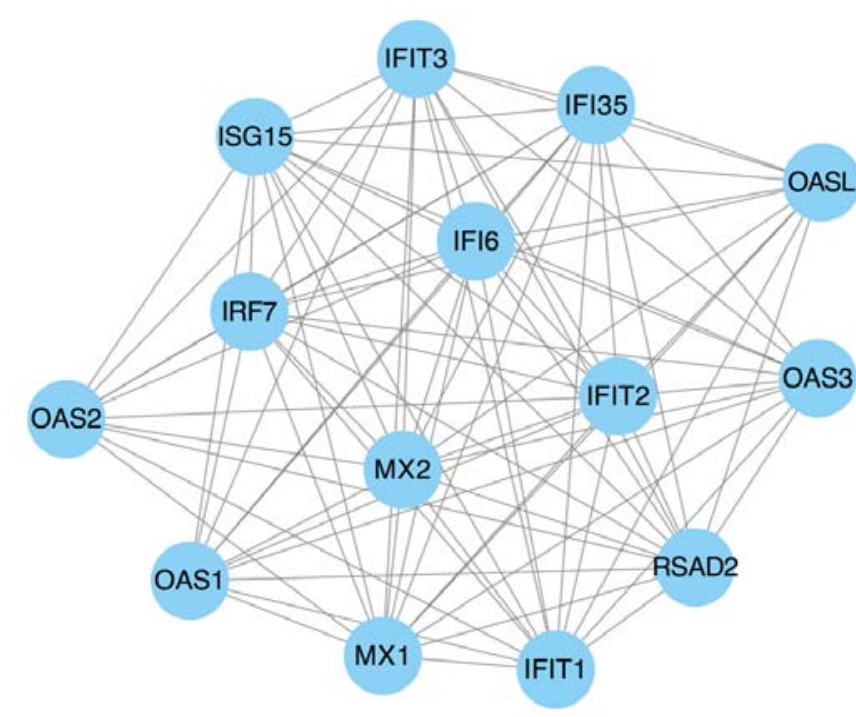

Figure 5. Identification of a sub-network using MCODE in Cytoscape software. $\mathrm{P}<0.05$

3-kinase catalytic subunit $\alpha(31,32)$, which indicates that miR-375 may be associated with the PI3K-Akt signaling pathway. Furthermore, survival analysis of the top 10 hub genes was performed using the publicly available database Kaplan-Meier Plotter. The Kaplan-Meier curves indicated 
JUN

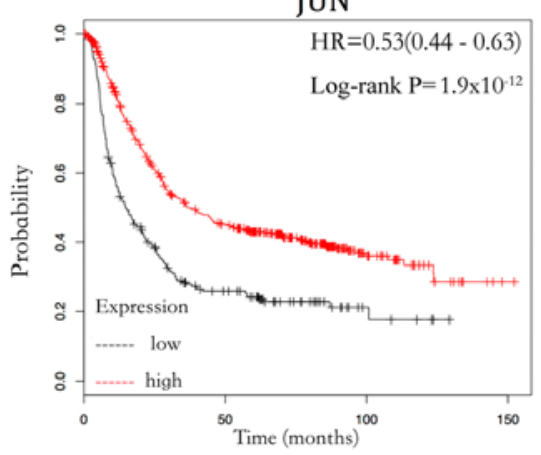

Number at risk
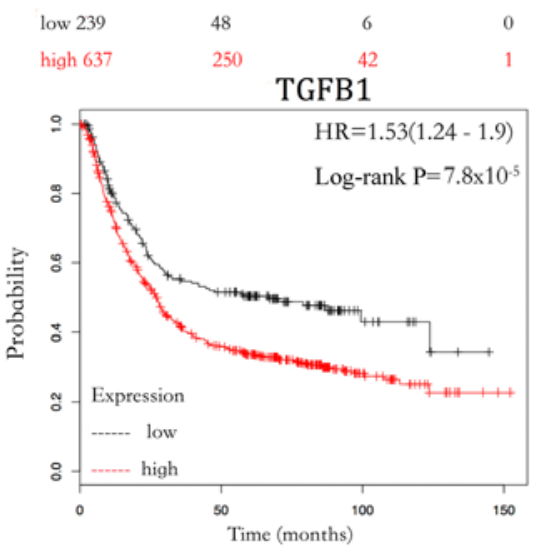

Number at risk
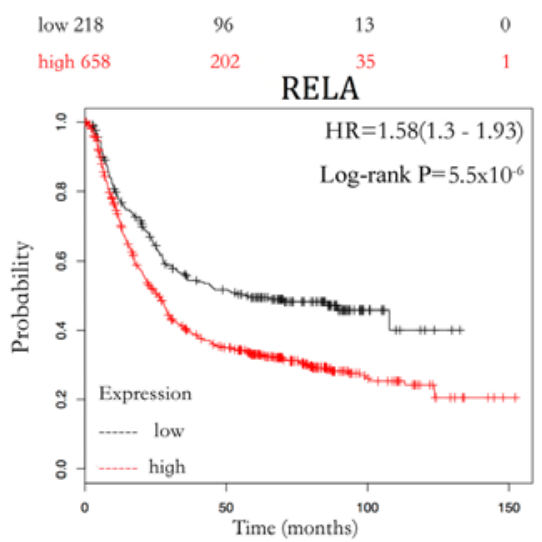

Number at risk

low $254 \quad 114$

high 622

VEGFA

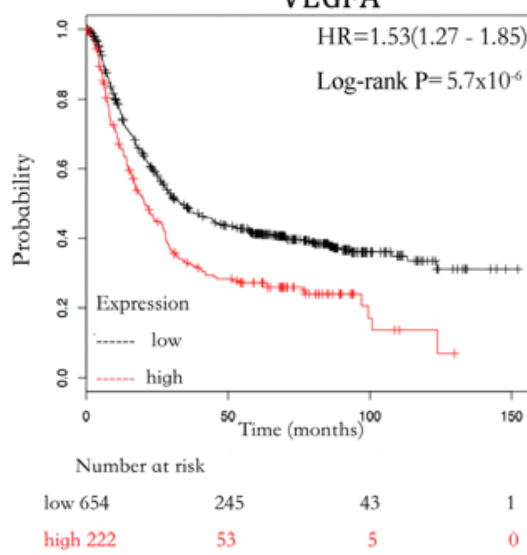

MAPK3

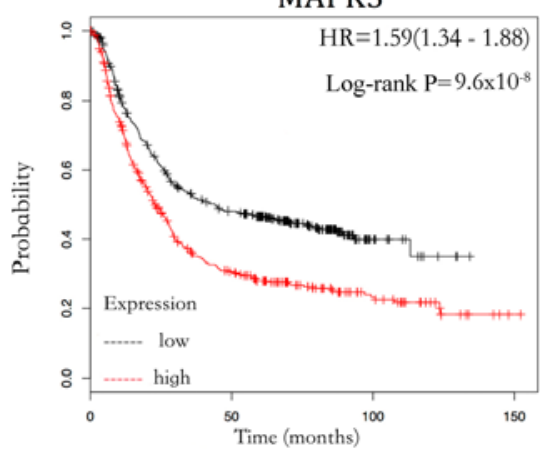

Number at risk
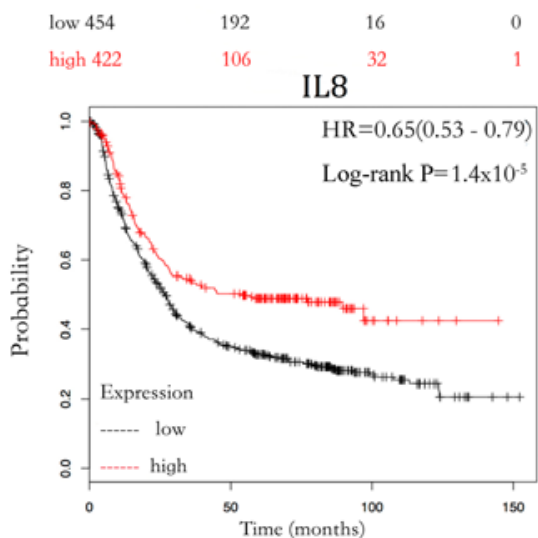

Number at risk
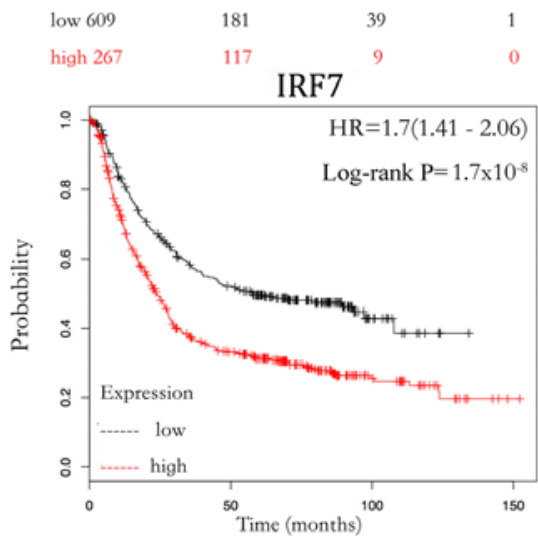

Number at risk

low $310 \quad 142$

high 566

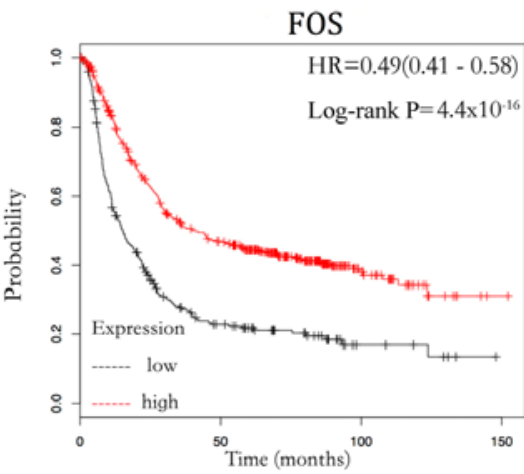

Number at risk

low $262 \quad 44$

high $614 \quad 254$

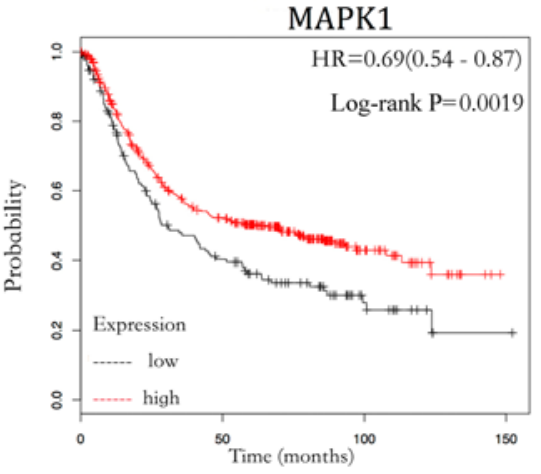

Number at risk

low $158 \quad 52$

high $473 \quad 213$

ISG15

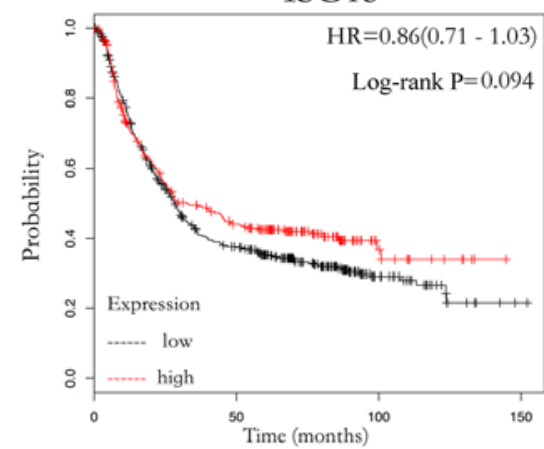

Number at risk

low $573 \quad 184$

high $303 \quad 114$

Figure 6. Survival analysis of 10 top hub genes. The survival data for patients with gastric cancer were obtained from Kaplan-Meier Plotter database. P $<0.05$. HR, hazard ratio; JUN, Jun proto-oncogene; TGFB1, transforming growth factor- $\beta 1$; FOS, Fos proto-oncogene, AP- 1 transcription factor subunit; IL- 8 , interleukin 8; ubiquitin like modifier (ISG15); MAPK, mitogen-activated protein kinase; RELA, RELA proto-oncogene NF- $\kappa$ subunit; IRF7, interferon regulatory factor 7; VEGFA, vascular endothelial growth factor A. 
Table IV. Enriched Kyoto Encyclopedia of Genes and Genomes pathway analysis of 158 differentially expressed mRNAs.

\begin{tabular}{|c|c|c|c|}
\hline Term & Count & P-value & Genes \\
\hline $\begin{array}{l}\text { bta04151:Phosphoinositide-3- } \\
\text { kinase-protein kinase B } \\
\text { signaling pathway }\end{array}$ & 10 & $6.79 \times 10^{-04}$ & $\begin{array}{l}\text { COL4A1, SGK2, COL6A3, COL1A2, } \\
\text { COL1A1, THBS1, COL11A1, THBS2, FN1, SPP1 }\end{array}$ \\
\hline bta04510:Focal adhesion & 9 & $9.84 \times 10^{-05}$ & $\begin{array}{l}\text { COL4A1, COL6A3, COL1A2, COL1A1, } \\
\text { THBS1, COL11A1, THBS2, FN1, SPP1 }\end{array}$ \\
\hline bta05146:Amoebiasis & 6 & 0.001142 & $\begin{array}{l}\text { COL4A1, COL1A2, CXCL8, } \\
\text { COL1A1, COL11A1, FN1 }\end{array}$ \\
\hline bta05144:Malaria & 3 & 0.054453 & CXCL8, THBS1, THBS2 \\
\hline bta01100:Metabolic pathways & 16 & 0.024433 & $\begin{array}{l}\text { CYP3A5, PIK3C2G, CYP2C18, ACER2, } \\
\text { ADH1C, ADH7, FBP2, AMPD1, ALDH3A1, } \\
\text { RDH12, AKR1B10, MGAM, TPH1, SMPD3, LIPF }\end{array}$ \\
\hline $\begin{array}{l}\text { bta04512:Extracellular } \\
\text { matrix-receptor interaction }\end{array}$ & 9 & $1.47 \times 10^{-07}$ & $\begin{array}{l}\text { COL4A1, COL6A3, COL1A2, COL1A1, } \\
\text { THBS1, COL11A1, THBS2, FN1, SPP1 }\end{array}$ \\
\hline bta04971:Gastric acid secretion & 7 & $1.07 \times 10^{-05}$ & $\begin{array}{l}\text { KCNJ16, KCNJ15, ATP4A, ATP4B, } \\
\text { SLC26A7, KCNE2, CA2 }\end{array}$ \\
\hline $\begin{array}{l}\text { bta04974:Protein digestion } \\
\text { and absorption }\end{array}$ & 7 & $2.61 \times 10^{-05}$ & $\begin{array}{l}\text { COL4A1, COL6A } 3, \text { COL1A2, COL12A1, } \\
\text { COL1A1, COL11A1, COL10A1 }\end{array}$ \\
\hline bta00830:Retinol metabolism & 6 & $4.79 \times 10^{-05}$ & RDH12, CYP3A5, CYP2C18, ADH1C, ADH7 \\
\hline bta05204:Chemical carcinogenesis & 6 & $1.29 \times 10^{-04}$ & CYP3A5, CYP2C18, ADH1C, ADH7, ALDH3A1 \\
\hline bta00140:Steroid hormone biosynthesis & 4 & 0.007616 & CYP3A5, STS, CYP2C18 \\
\hline bta00010:Glycolysis/Gluconeogenesis & 4 & 0.010031 & ADH1C, ADH7, FBP2, ALDH3A1 \\
\hline bta04966:Collecting duct acid secretion & 3 & 0.015591 & ATP4A, ATP4B, CA2 \\
\hline bta00591:Linoleic acid metabolism & 3 & 0.028276 & CYP3A5, CYP2C18 \\
\hline bta00350:Tyrosine metabolism & 3 & 0.032672 & ADH1C, ADH7, ALDH3A1 \\
\hline $\begin{array}{l}\text { bta00982:Drug metabolism- } \\
\text { cytochrome P450 }\end{array}$ & 3 & 0.063878 & ADH1C, ADH7, ALDH3A1 \\
\hline $\begin{array}{l}\text { bta00980:Metabolism of xenobiotics } \\
\text { by cytochrome P450 }\end{array}$ & 3 & 0.065826 & ADH1C, ADH7, ALDH3A1 \\
\hline
\end{tabular}

Table V. Four enriched Kyoto Encyclopedia of Genes and Genomes pathway analysis of 10 hub genes that are identical to the results of 158 differentially expressed mRNAs.

\begin{tabular}{lccl}
\hline Term & Count & P-value & Genes \\
\hline $\begin{array}{l}\text { bta04151:Phosphoinositide-3- } \\
\text { kinase-protein kinase B signaling pathway }\end{array}$ & 6 & $2.16 \times 10^{-05}$ & MAPK1, IL6, RELA, VEGFA, MAPK3 \\
bta04510:Focal adhesion & 4 & 0.001531 & MAPK1, JUN, VEGFA, MAPK3 \\
bta05146:Amoebiasis & 3 & 0.007335 & IL6, RELA, TGFB1 \\
bta05144:Malaria & 2 & 0.061465 & IL6, TGFB1
\end{tabular}

MAPK, mitogen-activated protein kinase; IL-6, interleukin 6; RELA, RELA proto-oncogene NF- $\kappa \beta$ subunit; VEGFA, vascular endothelial growth factor A; JUN, Jun proto-oncogene; TGFB1, transforming growth factor- $\beta 1$.

that the higher expression of MAPK3, TGFB1, RELA, IRF7 and VEGFA were associated with poor survival in GC. By contrast, a lower expression of JUN, FOS, IL8, ISG15 and MAPK1 were associated with poor survival in GC. Similar to the present study, a previous study applied bioinformatics analysis to identify the key genes in NCI-N87 GC cells exposed to quercetin. A PPI network was constructed, and hub genes were identified according to degree level, including FOS (degree=12) and JUN (degree=11) (33). One genome-wide search was performed to identify the genes epigenetically silenced by $\mathrm{CpG}$ methylation in GC. Three GC cell lines (SNU-1, SNU-601 and SNU-719) treated with 5Aza-Dc were analyzed to identify 143 associated genes by microarrays. Among the associated genes, IRF7 exhibited 
A

COL8A1

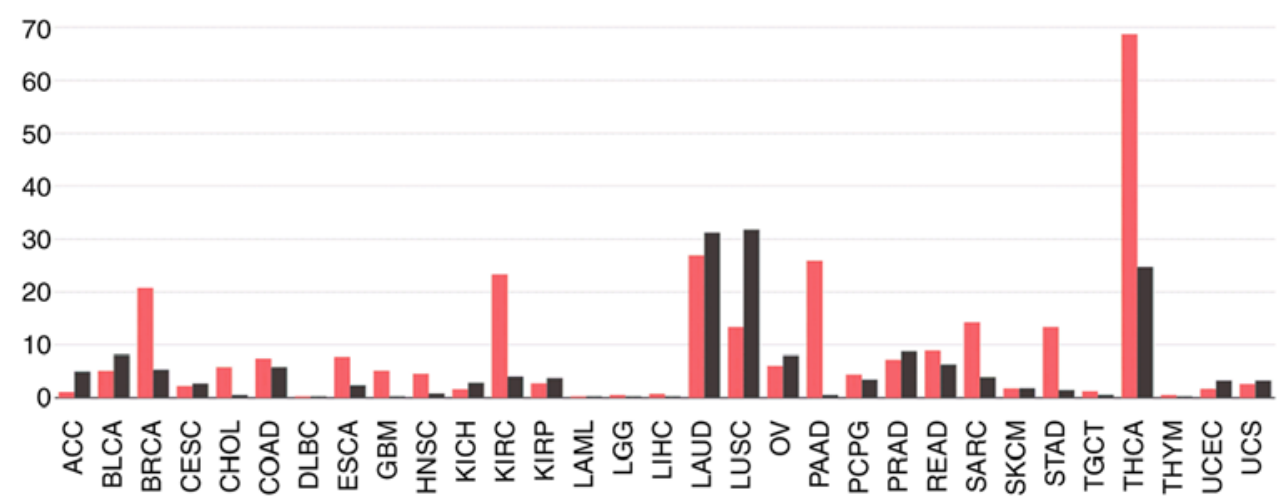

INHBA
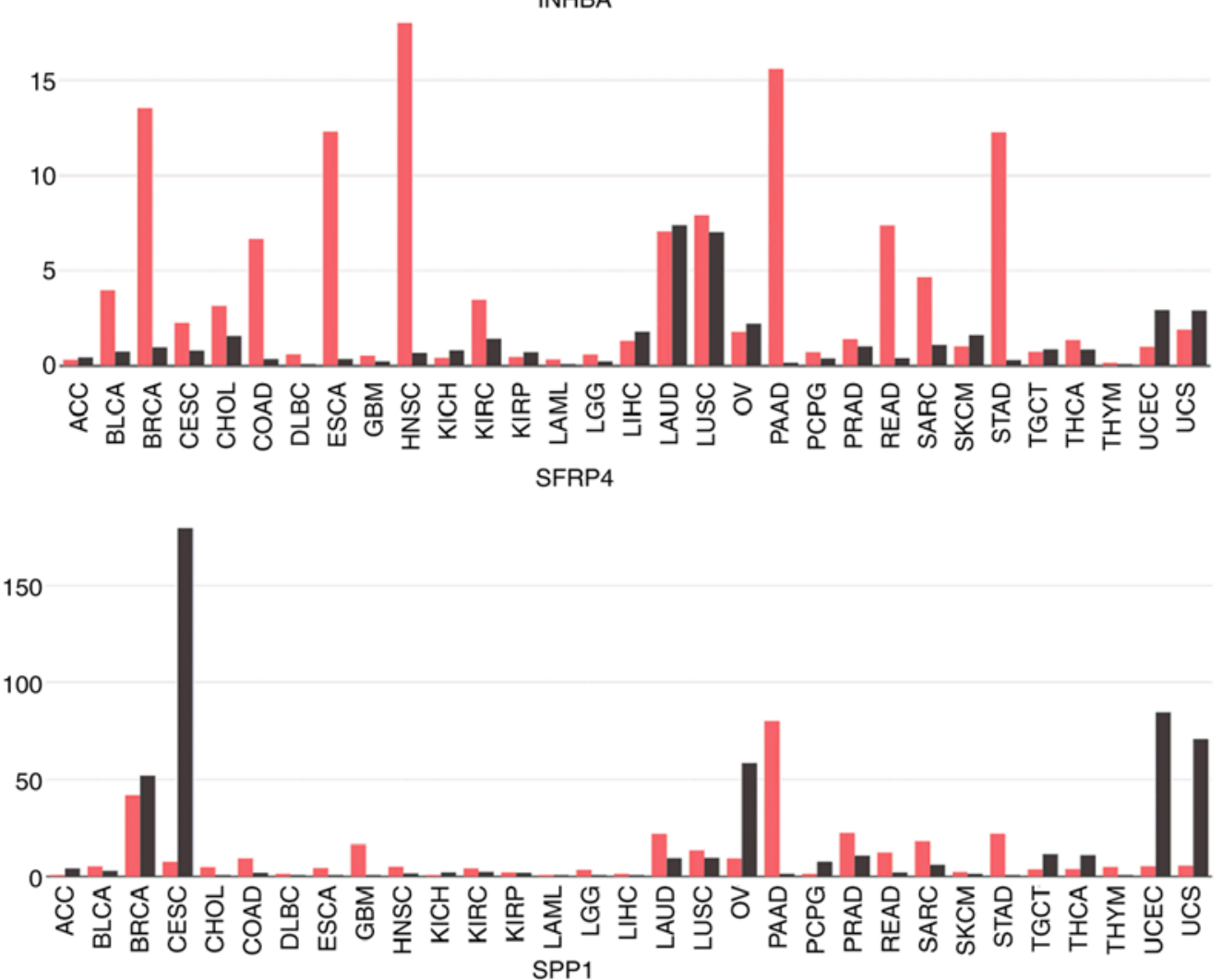

ㅁ. Tumor

- Normal

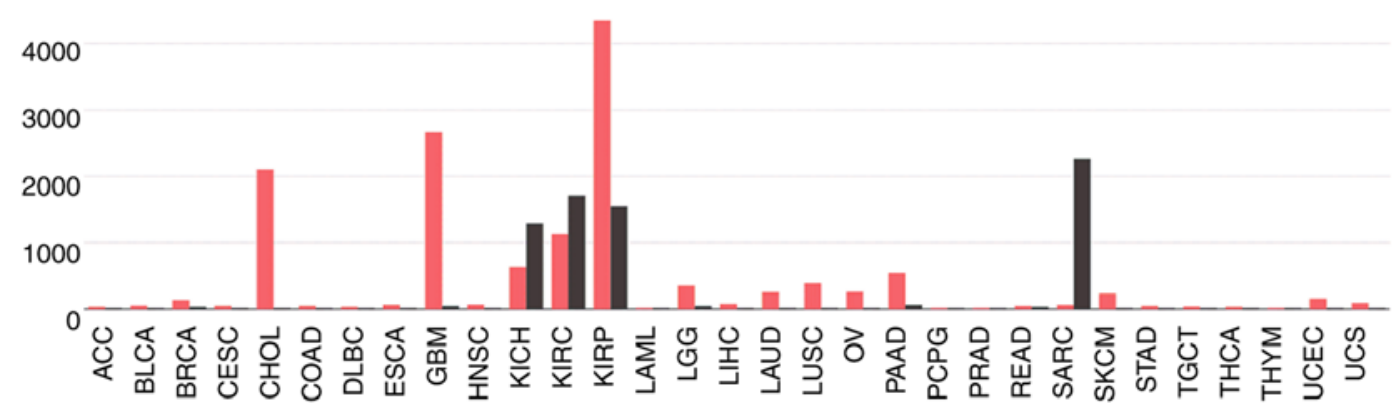

THBS2

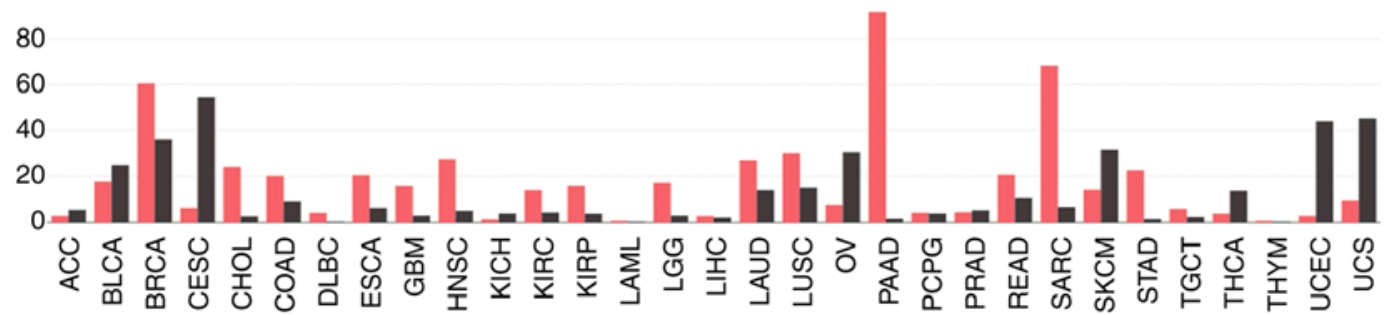

Figure 7. Expression levels of 10 DEMs in gastric cancer tissue from the GEPIA database. (A) 5 upregulated DEMs. 
B
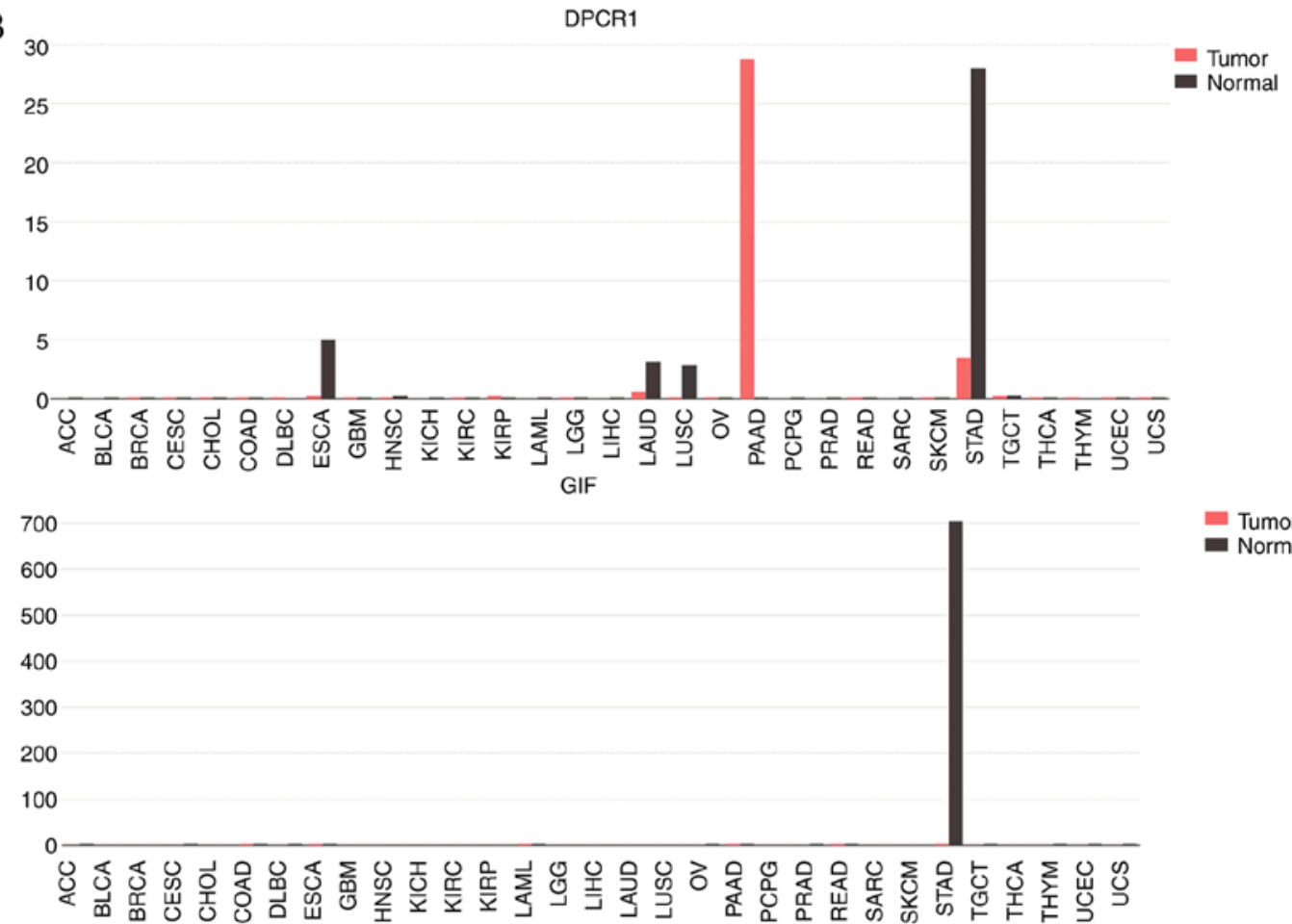

Tumor

GKN1

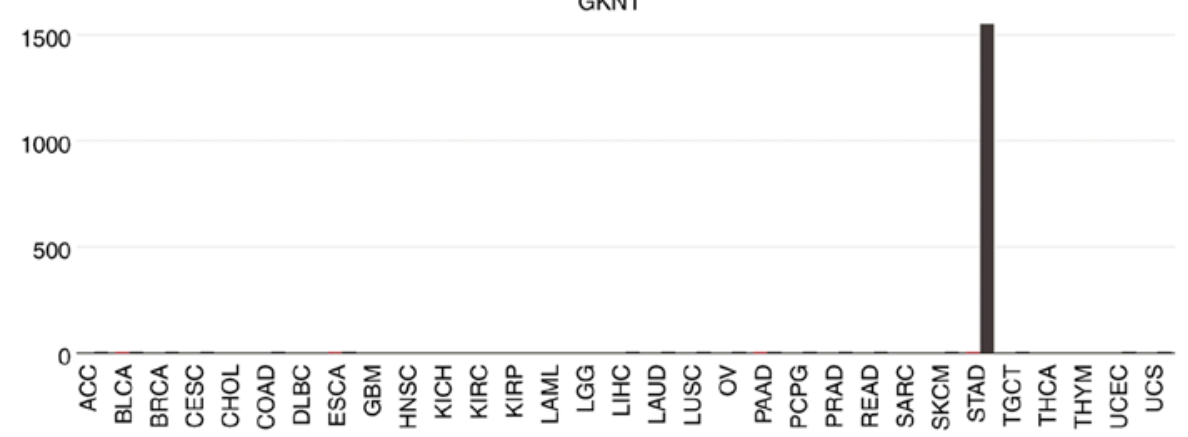

GKN2

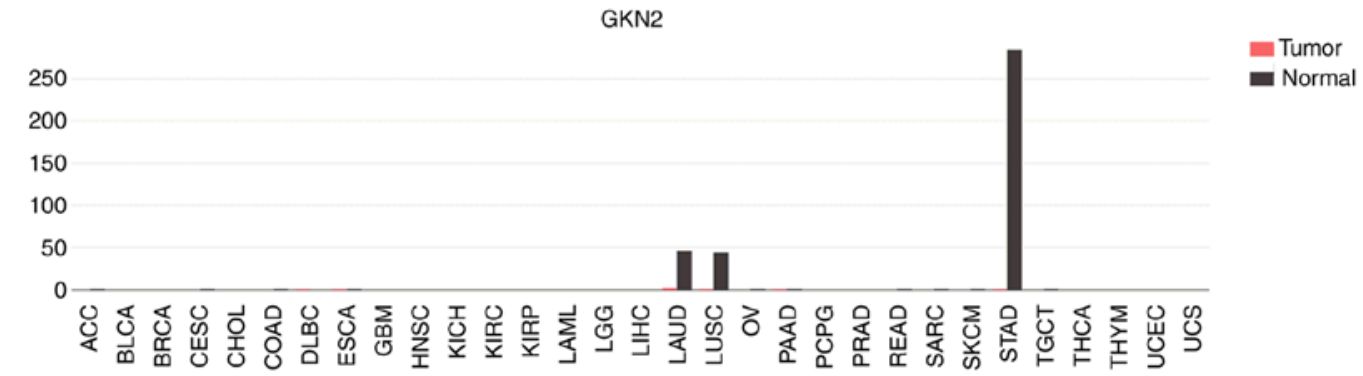

KRT20

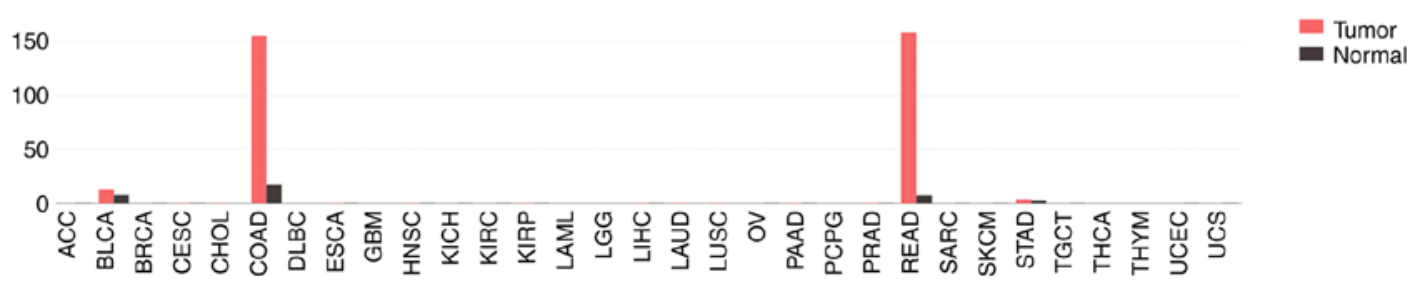

Figure 7. Continued. Expression levels of 10 DEMs in gastric cancer tissue from the GEPIA database. (B) 5 downregulated DEMs. P<0.05. DEM, differentially expressed mRNA.

promoter hypermethylation in one or more gastric cancer cell lines (34). As the intracellular signal transduction pathway, the MAPK cascade serves an important function in the progression of various tumor types. MAPK1 was upregulated in GC tissues and participated in the proliferation and cell migration of GC cells. In addition, miRNAs 
A
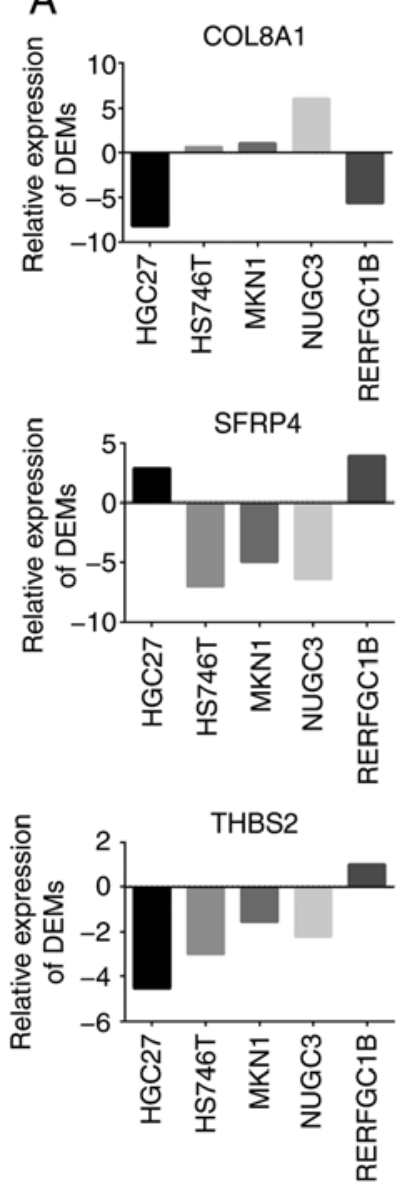
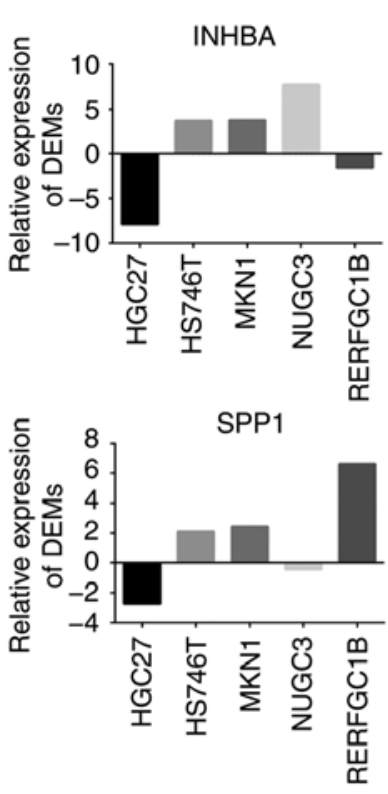
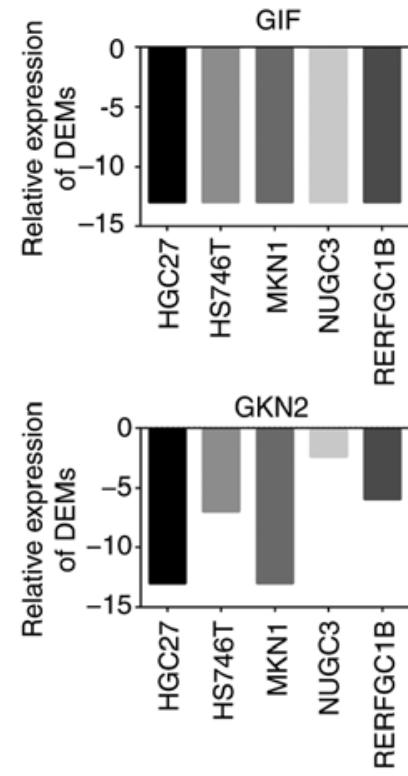

Figure 8. Expression levels of 10 DEMs in the Cancer Cell Line Encyclopedia database. (A) Expression levels of 5 upregulated DEMs in 5 GC cell lines. (B) Expression levels of 5 downregulated DEMs in 5 GC cell lines. P<0.05. DEM, differentially expressed mRNA; GC, gastric cancer.

may regulate the expression of MAPK1 and were therefore involved in the development and progression of GC $(35,36)$. In the analysis of patients with resected GC, the results of patient prognosis analysis indicated that MAPK3/1 expression was an independent prognostic marker for patients with resected GC (37). The NF- $\kappa \mathrm{B}$ signaling pathway serves an important function in the biological process of GC, including cell migration, cell invasion and cell apoptosis. As a basic component of the NF- $\kappa \mathrm{B}$ signaling pathway, RELA has been reported to be activated in the progression of GC. The results of a study by Huang et al (38) revealed that RELA was upregulated in GC tumor tissues and GC cell lines compared with control groups. Furthermore, the upregulation of RELA was significantly correlated with poor OS in the prognosis of 876 patients with $\mathrm{GC}(\mathrm{P}<0.001)$, which was consistent with the results of the present study. As an inflammatory factor, IL-6 serves an important function in the process of various types of cancer. Previous studies have revealed that IL-6 is involved in the regulation of invasion and prognosis in GC $(39,40)$. Mechanistically, mesenchymal stem cells promote the activation of neutrophils through the IL-6-STAT3 axis to mediate GC progression (41). IL8 has been reported to be involved in the development of GC. A previous study demonstrated that IL- 8 was upregulated in GC cells and enhanced cell proliferation, invasion and migration in GC (42). In addition, IL8 served as an inflam-
B
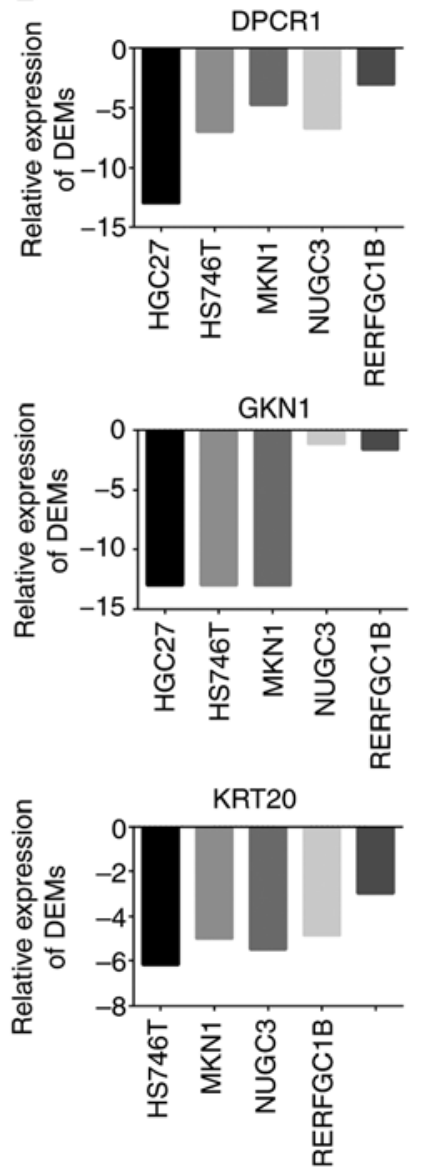

matory cytokine serving an important function in the angiogenesis of GC (43). Furthermore, a previous study also conducted a meta-analysis to investigate the potential functions of IL-6 and IL-8 polymorphisms in the development of GC. The results revealed that IL-6 rs1800796 and IL-8 rs4073 polymorphisms may serve as genetic biomarkers of GC in an Asian population (44). A number of studies have indicated that VEGFA functions as a direct target of miRNAs to participate in the tumor progression of GC $(45,46)$. Furthermore, VEGFA may also be a predictive biomarker for antiangiogenic therapy in GC, which is consistent with the present bioinformatics results (47). A previous study also conducted analysis to reveal the correlation between polymorphisms of TGFB1 and VEGF genes and the survival of patients with GC. The results suggested that the TGFB1+915CG/CC and VEGF-634CG genotypes were associated with short-term survival in patients with GC (48).

MiRNAs serve an important function in the downregulation of the transcription of target mRNAs by binding to complementary 3'-untranslated regions of genes (49). An accumulating body of evidence has demonstrated the association between miRNAs and targets in tumor progression in various types of cancer. For example, it has been confirmed that miR-218 is a tumor suppressor in glioblastoma cells by directly targeting E2F transcription factor 2 (50). miR-155 


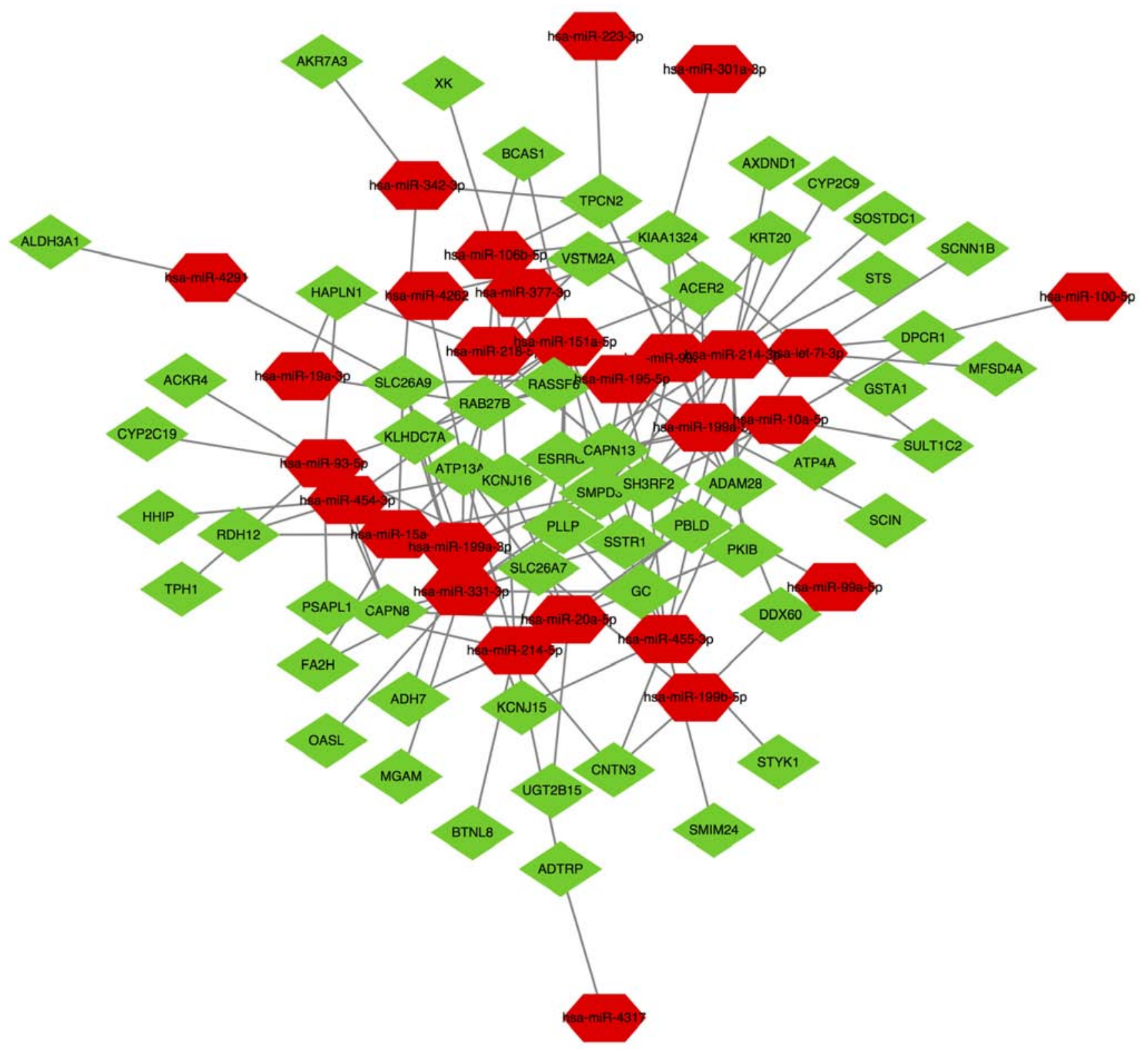

Figure 9. Network of microRNAs and targets. $\mathrm{P}<0.05$.

has also been demonstrated to enhance cell growth and invasion by regulating transforming growth factor- $\beta$ receptor 2 (51). In the present study, a total of 30 upregulated miRNAs and 1 downregulated miRNA were identified between the GC and control samples using systematic analysis. Then, a network of miRNAs and targets was constructed using the miRwalk database and Cytoscape software, which consisted of 124 nodes and 490 interactions. According to the results of the present analysis, miR-375 was downregulated in GC tissues when compared with the NC group. Similarly, a number of studies have confirmed the function of miR-375 as a tumor suppressor in GC. MiR-375 has been reported to be involved in cell proliferation, migration and invasion by regulating janus kinase 2 and macrophage stimulating 1 receptor $(52,53)$. MiR-375 serves as a controller of the Hippo signaling pathway by targeting the Yes associated protein 1-TEA domain transcription factor 4-cellular communication network factor 2 axis (54). A previous study indicated that miR19a3p is upregulated in GC and promotes epithelialmesenchymal transition via the PI3K/Akt signaling pathway (55). Furthermore, miR-19a-3P has been confirmed to mediate metastasis by directly targeting MAX dimerization protein 1 in GC cells (56). Emerging evidence has indicated that miR-214-3p serves an essential oncogenic function and is correlated with distant metastasis as a novel biomarker of GC (57). In addition, miR-214-3p negatively regulates phosphatase and tensin homolog and is involved in GC cell proliferation, migration and invasion $(58,59)$.

Previous studies have suggested that lncRNAs participate in the development of cell growth, metastasis and invasion progression in various types of cancer (60-62). LncRNAs have also been demonstrated to function as miRNA sponges that are involved in a variety of cancer types. For example, the IncRNA XIST has been demonstrated to function as a molecular sponge of miR-101 to modulate enhancer of zeste 2 polycomb repressive complex 2 subunit expression in GC (63). 


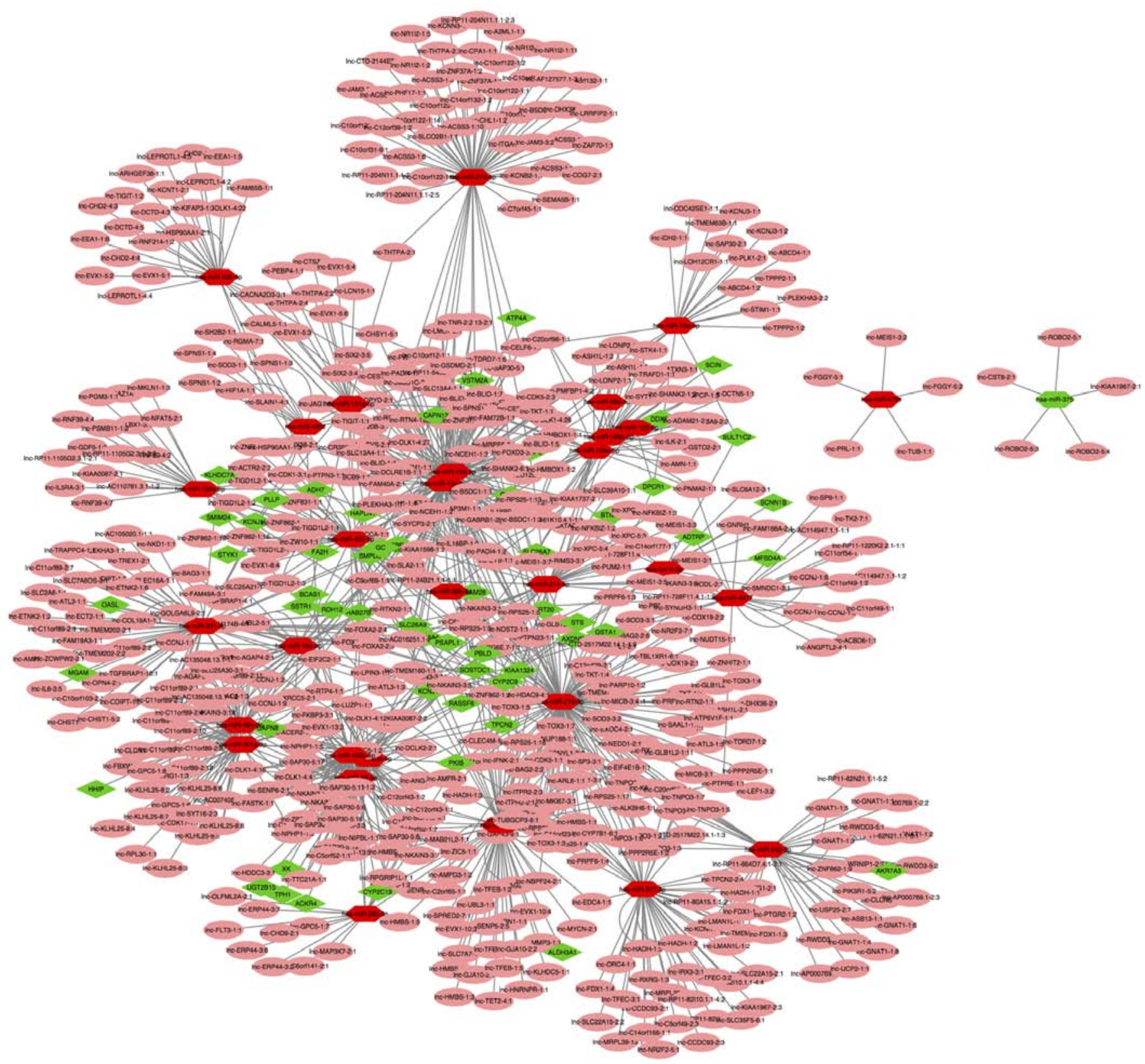

Figure 10. LncRNA-miRNA-mRNA regulatory network. Pink circles indicate lncRNAs, hexagons indicate miRNAs and diamonds represent mRNAs. Red nodes indicate upregulated mRNAs and miRNAs, and green nodes represent the downregulated mRNAs and miRNAs. P $<0.05$. LncRNA, long non-coding RNA; miRNA, microRNA.

LncRNA-regulator of reprogramming regulates the expression of miR-145 and ADP ribosylation factor 6 by functioning as a sponge in triple-negative breast cancer (64). Therefore, it is essential to investigate the regulatory mechanism underlying the action of lncRNAs, miRNAs and mRNAs. In the present study, the lncRNASNP database was used to perform prediction analysis of DEMis-lncRNA pairs. In addition, a lncRNA-miRNA-mRNA network was constructed using the combination of DEMis-target pairs and DEMis-lncRNA pairs, which included 1,215 regulatory associations. Altogether, these results provide a better understanding of the potential functions of lncRNA, miRNA and mRNA in GC.

However, there were a number of limitations in the present analysis. Firstly, the present study lacked experimental evidence on the expression levels and biological functions of genes. Further detection and experiments on the associated genes in larger sample sizes should be validated in future studies. Secondly, lncRNAs were only predicted using the lncRNASNP database without the investigation of expression profile databases, which are different from mRNAs and miRNAs. In order to increase the credibility, GC-associated IncRNAs require further analysis in the future.

In conclusion, the present study analyzed 158 DEMs, 31 DEMis and the top 10 hub genes in GC from multiple profile datasets by integrated bioinformatical analysis. In addition, lncRNA-miRNA-mRNA regulatory networks were established to gain a better understanding of the underlying molecular mechanism of GC. These results provide an effective foundation for further research on potential target therapy strategies in GC. 


\section{Acknowledgements}

Not applicable.

\section{Funding}

The present study was supported in part by the Department of Gastrointestinal Endoscopy, First Affiliated Hospital of China Medical University (Shenyang, China).

\section{Availability of data and materials}

The datasets used and/or analyzed during the present study are available from the corresponding author on reasonable request.

\section{Authors' contributions}

MS designed the study. XM, YM, HZ and HJZ performed the research. XM analyzed the data and wrote the paper.

\section{Ethics approval and consent to participate}

Not applicable

\section{Patient consent for publication}

Not applicable

\section{Competing interests}

The authors declare that they have no competing interests.

\section{References}

1. Siegel RL, Miller KD and Jemal A: Cancer statistics, 2016. CA Cancer J Clin 66: 7-30, 2016.

2. Karimi P, Islami F, Anandasabapathy S, Freedman ND and Kamangar F: Gastric cancer: Descriptive epidemiology, risk factors, screening, and prevention. Cancer Epidemiol Biomarkers Prev 23: 700-713, 2014.

3. Wang J, Guo W, Wu Q, Zhang R and Fang J: Impact of combination epidural and general anesthesia on the long-term survival of gastric cancer patients: A retrospective study. Med Sci Monit 22: 2379-2385, 2016.

4. Long J, Zhang Z, Liu Z, Xu Y and Ge C: Identification of genes and pathways associated with pancreatic ductal adenocarcinoma by bioinformatics analyses. Oncol Lett 11: 1391-1397, 2016.

5. He JH, Han ZP, Zou MX, Wang L, Lv YB, Zhou JB, Cao MR and Li YG: Analyzing the LncRNA, miRNA, and mRNA regulatory network in prostate cancer with bioinformatics software. J Comput Biol 25: 146-157, 2018.

6. Wang H, Luo J, Liu C, Niu H, Wang J, Liu Q, Zhao Z, Xu H, Ding Y, Sun J and Zhang Q: Investigating MicroRNA and transcription factor co-regulatory networks in colorectal cancer. BMC Bioinformatics 18: 388, 2017.

7. Guo Y, Bao Y, Ma M and Yang W: Identification of key candidate genes and pathways in colorectal cancer by integrated bioinformatical analysis. Int J Mol Sci 18: pii: E722, 2017.

8. Piao J, Sun J, Yang Y, Jin T, Chen L and Lin Z: Target gene screening and evaluation of prognostic values in non-small cell lung cancers by bioinformatics analysis. Gene 647: 306-311, 2018.

9. Jiang H, Ma R, Zou S, Wang Y, Li Z and Li W: Reconstruction and analysis of the lncRNA-miRNA-mRNA network based on competitive endogenous RNA reveal functional lncRNAs in rheumatoid arthritis. Mol Biosyst 13: 1182-1192, 2017.

10. Edgar R, Domrachev M and Lash AE: Gene expression omnibus: NCBI gene expression and hybridization array data repository Nucleic Acids Res 30: 207-210, 2002.
11. Pathan M, Keerthikumar S, Ang CS, Gangoda L, Quek CY, Williamson NA, Mouradov D, Sieber OM, Simpson RJ, Salim A, et al: FunRich: An open access standalone functional enrichment and interaction network analysis tool. Proteomics 15: 2597-2601, 2015.

12. Davis S and Meltzer PS: GEOquery: A bridge between the gene expression omnibus (GEO) and BioConductor. Bioinformatics 23: 1846-1847, 2007.

13. Szklarczyk D, Gable AL, Lyon D, Junge A, Wyder S, Huerta-Cepas J, Simonovic M, Doncheva NT, Morris JH, Bork P, et al: STRING v11: Protein-protein association networks with increased coverage, supporting functional discovery in genome-wide experimental datasets. Nucleic Acids Res 47 (D1): D607-D613, 2019.

14. Shannon P, Markiel A, Ozier O, Baliga NS, Wang JT, Ramage D, Amin N, Schwikowski B and Ideker T: Cytoscape: A software environment for integrated models of biomolecular interaction networks. Genome Res 13: 2498-2504, 2003.

15. Bader GD and Hogue CW: An automated method for finding molecular complexes in large protein interaction networks. BMC Bioinformatics 4: 2, 2003.

16. Nagy Á, Lánczky A, Menyhárt O and Győrffy B: Validation of miRNA prognostic power in hepatocellular carcinoma using expression data of independent datasets. Sci Rep 8: 9227, 2018.

17. Tang Z, Li C, Kang B, Gao G, Li C and Zhang Z: GEPIA: A web server for cancer and normal gene expression profiling and interactive analyses. Nucleic Acids Res 45 (W1): W98-W102, 2017.

18. Ghandi M, Huang FW, Jané-Valbuena J, Kryukov GV, Lo CC, McDonald ER III, Barretina J, Gelfand ET, Bielski CM, $\mathrm{Li} \mathrm{H}$, et al: Next-generation characterization of the cancer cell line encyclopedia. Nature 569: 503-508, 2019.

19. Dweep H, Sticht C, Pandey P and Gretz N: miRWalk-database: Prediction of possible miRNA binding sites by 'walking' the genes of three genomes. J Biomed Inform 44: 839-847, 2011.

20. Dweep H and Gretz N: miRWalk2.0: A comprehensive atlas of microRNA-target interactions. Nat methods 12: 697, 2015.

21. Miao YR, Liu W, Zhang Q and Guo AY: lncRNASNP2: An updated database of functional SNPs and mutations in human and mouse lncRNAs. Nucleic Acids Res 46 (D1): D276-D280, 2018.

22. He J, Jin Y, Chen Y, Yao HB, Xia YJ, Ma YY, Wang W and Shao QS: Downregulation of ALDOB is associated with poor prognosis of patients with gastric cancer. Onco Targets Ther 9: 6099-6109, 2016.

23. Qi W, Sun L, Liu N, Zhao S, Lv J and Qiu W: Tetraspanin family identified as the central genes detected in gastric cancer using bioinformatics analysis. Mol Med Rep 18: 3599-3610, 2018.

24. Liu Q, Dong HW, Sun WG, Liu M, Ibla JC, Liu LX, Parry JW, Han XH, Li MS and Liu JR: Apoptosis initiation of $\beta$-ionone in SGC-7901 gastric carcinoma cancer cells via a PI3K-AKT pathway. Arch Toxicol 87: 481-490, 2013.

25. Li GQ, Xie J, Lei XY and Zhang L: Macrophage migration inhibitory factor regulates proliferation of gastric cancer cells via the PI3K/Akt pathway. World J Gastroenterol 15: 5541-5548, 2009.

26. Ye B, Jiang LL, Xu HT, Zhou DW and Li ZS: Expression of PI3K/AKT pathway in gastric cancer and its blockade suppresses tumor growth and metastasis. Int J Immunopathol Pharmacol 25: 627-636, 2012.

27. Ang KL, Shi DL, Keong WW and Epstein RJ: Upregulated Akt signaling adjacent to gastric cancers: Implications for screening and chemoprevention. Cancer Lett 225: 53-59, 2005.

28. Xing CG, Zhu BS, Fan XQ, Liu HH, Hou X, Zhao K and Qin ZH: Effects of LY294002 on the invasiveness of human gastric cancer in vivo in nude mice. World J Gastroenterol 15: 5044-5052, 2009.

29. Zhang K, Zhong W, Li WP, Chen ZJ and Zhang C: miR-15a-5p levels correlate with poor ovarian response in human follicular fluid. Reproduction 154: 483-496, 2017.

30. Sun W, Li Y and Wei S: miR-4262 regulates chondrocyte viability, apoptosis, autophagy by targeting SIRT1 and activating $\mathrm{PI} 3 \mathrm{~K} / \mathrm{AKT} / \mathrm{mTOR}$ signaling pathway in rats with osteoarthritis. Exp Ther Med 15: 1119-1128, 2018.

31. Shi ZC, Chu XR, Wu YG, Wu JH, Lu CW, Lü RX, Ding MC and Mao NF: MicroRNA-375 functions as a tumor suppressor in osteosarcoma by targeting PIK3CA. Tumour Biol 36: 8579-8584, 2015.

32. Wang Y, Tang Q, Li M, Jiang S and Wang X: MicroRNA-375 inhibits colorectal cancer growth by targeting PIK3CA. Biochem Biophys Res Commun 444: 199-204, 2014. 
33. Zeng Y, Shen Z, Gu W and Wu M: Bioinformatics analysis to identify action targets in NCI-N87 gastric cancer cells exposed to quercetin. Pharm Biol 56: 393-398, 2018.

34. Jee CD, Kim MA, Jung EJ, Kim J and Kim WH: Identification of genes epigenetically silenced by $\mathrm{CpG}$ methylation in human gastric carcinoma. Eur J Cancer 45: 1282-1293, 2009.

35. Xiong HL, Zhou SW, Sun AH, He Y, Li J and Yuan X: MicroRNA-197 reverses the drug resistance of fluorouracil-induced SGC7901 cells by targeting mitogen-activated protein kinase 1. Mol Med Rep 12: 5019-5025, 2015.

36. Hu L, Wu H, Wan X, Liu L, He Y, Zhu L, Liu S, Yao H3 and Zhu Z: MicroRNA-585 suppresses tumor proliferation and migration in gastric cancer by directly targeting MAPK1. Biochem Biophys Res Commun 499: 52-58, 2018.

37. Kim JG, Lee SJ, Chae YS, Kang BW, Lee YJ, Oh SY, Kim MC Kim KH and Kim SJ: Association between phosphorylated AMP-activated protein kinase and MAPK3/1 expression and prognosis for patients with gastric cancer. Oncology 85: 78-85, 2013.

38. Huang T, Kang W, Zhang B, Wu F, Dong Y, Tong JH, Yang W, Zhou Y, Zhang L, Cheng AS, et al: miR-508-3p concordantly silences NFKB1 and RELA to inactivate canonical NF- $\mathrm{KB}$ signaling in gastric carcinogenesis. Mol Cancer 15: 9, 2016.

39. Xia Y, Khoi PN, Yoon HJ, Lian S, Joo YE, Chay KO, Kim KK and Jung YD: Piperine inhibits IL-1 $\beta$-induced IL- 6 expression by suppressing p38 MAPK and STAT3 activation in gastric cancer cells. Mol Cell Biochem 398: 147-156, 2015.

40. Yin Y, Si X, Gao Y, Gao L and Wang J: The nuclear factor- $\kappa \mathrm{B}$ correlates with increased expression of interleukin- 6 and promotes progression of gastric carcinoma. Oncol Rep 29: 34-38, 2013.

41. Zhu Q, Zhang X, Zhang L, Li W, Wu H, Yuan X, Mao F, Wang M, Zhu W, Qian $\mathrm{H}$ and Xu W: The IL-6-STAT3 axis mediates a reciprocal crosstalk between cancer-derived mesenchymal stem cells and neutrophils to synergistically prompt gastric cancer progression. Cell Death Dis 5: e1295, 2014.

42. Li W, Lin S, Li W, Wang W, Li X and Xu D: IL-8 interacts with metadherin promoting proliferation and migration in gastric cancer. Biochem Biophys Res Commun 478: 1330-1337, 2016.

43. Shi J, Li YJ, Yan B and Wei PK: Interleukin-8: A potent promoter of human lymphatic endothelial cell growth in gastric cancer. Oncol Rep 33: 2703-2710, 2015.

44. Wang X, Yang F, Xu G and Zhong S: The roles of IL-6, IL-8 and IL-10 gene polymorphisms in gastric cancer: A meta-analysis. Cytokine 111: 230-236, 2018.

45. Wang CQ, Chen L, Dong CL, Song Y, Shen ZP, Shen WM and Wu XD: MiR-377 suppresses cell proliferation and metastasis in gastric cancer via repressing the expression of VEGFA. Eur Rev Med Pharmacol Sci 21: 5101-5111, 2017

46. Zhang X, Tang J, Zhi X, Xie K, Wang W, Li Z, Zhu Y, Yang L, $\mathrm{Xu} \mathrm{H}$ and $\mathrm{Xu} \mathrm{Z}$ : Correction: miR-874 functions as a tumor suppressor by inhibiting angiogenesis through STAT3/VEGF-A pathway in gastric cancer. Oncotarget 8: 29535, 2017.

47. Caporarello N, Lupo G, Olivieri M, Cristaldi M, Cambria MT, Salmeri M and Anfuso CD: Classical VEGF, Notch and Ang signalling in cancer angiogenesis, alternative approaches and future directions (Review). Mol Med Rep 16: 4393-4402, 2017.

48. Guan X,Zhao H,Niu J, Tan D, Ajani JA and WeiQ: Polymorphisms of TGFB1 and VEGF genes and survival of patients with gastric cancer. J Exp Clin Cancer Res 28: 94, 2009.
49. Cho WC: OncomiRs: The discovery and progress of microRNAs in cancers. Mol Cancer 6: 60, 2007.

50. Zhang Y, Han D, Wei W, Cao W, Zhang R, Dong Q, Zhang J, Wang Y and Liu N: MiR-218 inhibited growth and metabolism of human glioblastoma cells by directly targeting E2F2. Cell Mol Neurobiol 35: 1165-1173, 2015.

51. Qu Y, Zhang H, Sun W, Han Y, Li S, Qu Y, Ying G and Ba Y: MicroRNA-155 promotes gastric cancer growth and invasion by negatively regulating transforming growth factor- $\beta$ receptor 2 . Cancer Sci 109: 618-628, 2018.

52. Ding L, Xu Y, Zhang W, Deng Y, Si M, Du Y, Yao H, Liu X, Ke Y, Si J and Zhou T: MiR-375 frequently downregulated in gastric cancer inhibits cell proliferation by targeting JAK2. Cell Res 20: 784-793, 2010

53. Lian S, Park JS, Xia Y, Nguyen TT, Joo YE, Kim KK, Kim HK and Jung YD: MicroRNA-375 functions as a tumor-suppressor gene in gastric cancer by targeting recepteur d'Origine nantais. Int J Mol Sci 17: pii: E1633, 2016.

54. Kang W, Huang T, Zhou Y, Zhang J, Lung RWM, Tong JHM, Chan AWH, Zhang B, Wong CC, Wu F, et al: miR-375 is involved in Hippo pathway by targeting YAP1/TEAD4-CTGF axis in gastric carcinogenesis. Cell Death Dis 9: 92, 2018.

55. Lu WD, Zuo Y, Xu Z and Zhang M: MiR-19a promotes epithelial-mesenchymal transition through PI3K/AKT pathway in gastric cancer. World J Gastroenterol 21: 4564-4573, 2015.

56. Wu Q, Yang Z, An Y, Hu H, Yin J, Zhang P, Nie Y, Wu K, Shi Y and Fan D: MiR-19a/b modulate the metastasis of gastric cancer cells by targeting the tumour suppressor MXD1. Cell Death Dis 5: e1144, 2014

57. Zhang KC, Xi HQ, Cui JX, Shen WS, Li JY, Wei B and Chen L: Hemolysis-free plasma miR-214 as novel biomarker of gastric cancer and is correlated with distant metastasis. Am J Cancer Res 5: 821-829, 2015.

58. Xin R, Bai F, Feng Y, Jiu M, Liu X, Bai F, Nie Y and Fan D: MicroRNA-214 promotes peritoneal metastasis through regulating PTEN negatively in gastric cancer. Clin Res Hepatol Gastroenterol 40: 748-754, 2016.

59. Yang TS, Yang XH, Wang XD, Wang YL, Zhou B and Song ZS MiR-214 regulate gastric cancer cell proliferation, migration and invasion by targeting PTEN. Cancer Cell Int 13: 68, 2013.

60. Prensner JR and Chinnaiyan AM: The emergence of lncRNAs in cancer biology. Cancer Discov 1: 391-407, 2011.

61. Rinn JL and Chang HY: Genome regulation by long noncoding RNAs. Annu Rev Biochem 81: 145-166, 2012.

62. Gutschner T and Diederichs S: The hallmarks of cancer: A long non-coding RNA point of view. RNA Biol 9: 703-719, 2012.

63. Chen DL, Ju HQ, Lu YX, Chen LZ, Zeng ZL, Zhang DS, Luo HY, Wang F, Qiu MZ, Wang DS, et al: Long non-coding RNA XIST regulates gastric cancer progression by acting as a molecular sponge of miR-101 to modulate EZH2 expression. J Exp Clin Cancer Res 35: 142, 2016.

64. Eades G, Wolfson B, Zhang Y, Li Q, Yao Y and Zhou Q: lincRNA-RoR and miR-145 regulate invasion in triple-negative breast cancer via targeting ARF6. Mol Cancer Res 13: 330-338, 2015 .

c) () $\Theta$ This work is licensed under a Creative Commons Attribution-NonCommercial-NoDerivatives 4.0 International (CC BY-NC-ND 4.0) License. 